Ann. Sci. forest., 1973, 30 (1), 1-29.

\title{
VARIATIONS SAISONNIÈRES ET STATIONNELLES DE LA TENEUR EN ÉLÉMENTS MINÉRAUX DES FEUILLES DE HÊTRE (FAGUS SYLVATICA) DANS L'EST DE LA FRANCE
}

\author{
F. LE TACON* et F. TOUTAIN** \\ avec la collaboration technique de Maryse BitsCH*, D. BouCHARD* \\ M. DorRISSE** et Mme P. JEANSON** \\ *Station de Recherches sur les Sols forestiers et la Fertilisation, \\ Centre national de Recherches forestières, I.N.R.A. \\ Champenoux 54370 Einville \\ **Centre de Pédologie Biologique, \\ Centre national de la Recherche scientifique \\ 54500 Vandouvre
}

\section{RÉSUMÉ}

Le hêtre, tout en étant une essence très plastique, est très influencé par les conditions de station, qui agissent soit sur la production de bois, soit sur sa qualité. A l'inverse, le hêtre n'est pas sans influence sur la pédogénèse par l'intermédiaire de la litière qu'il produit.

Il est très important de connaître les interrelations sol-hêtre, donc les conditions de nutrition minérale du hêtre, ainsi que les facteurs qui les régissent.

Nous avons étudié dans l'Est de la France la variation de la teneur en eau des feuilles et de leur teneur en divers éléments minéraux, d'une part en fonction du temps (prélèvements mensuels dans deux stations à sol très différent) et, d'autre part en fonction de la station (prélèvements effectués en août et en octobre dans 65 stations à sol très divers).

D'un point de vue méthodologique, en vue du diagnostic foliaire, il apparaît que le mois d'août est la meilleure période de prélèvement, en raison de l'équilibre existant à cette époque, dans les feuilles, entre les synthèses et les transferts organominéraux.

L'étude de la variation stationnelle met en relief l'importance du manganèse, du calcium et du fer dans la discrimination des stations, alors que l'azote présente une variabilité beaucoup plus faible.

Le manganèse et le fer pourraient ainsi jouer, en particulier dans les stations acides, un rôle important dans la formation des divers types d'humus et dans l'évolution du rapport $\mathrm{C} / \mathrm{N}$ de la litière, qui, au moment de la chute des feuilles, ne diffère que fort peu entre des stations pourtant très différentes. 


\section{INTRODUCTION}

Le hêtre est une essence très plastique, que l'on rencontre sous des climats très variés et dans des conditions de sols et d'humus extrêmement différentes.

Malgré cette variabilité assez étonnante, il existe une apparente uniformité des retombées biologiques. L'un d'entre nous (Toutain, Duchaufour 1970) a montré que le retour par les litières des éléments majeurs était très peu variable en poids, en comparaison de la gamme de composition des roches-mères.

Néanmoins, les effets des caractéristiques de la station sont considérables, tant sur la production que sur la qualité du bois. L'influence du type de station sur la production du hêtre est connue depuis longtemps à la suite des nombreux travaux belges (GALOUx 1954, Reginster 1955, DaGnelie 1956-1957, Manil et alt. 1963).

En ce qui concerne les caractéristiques du bois, il semble que les conditions de sols aient une importance non négligeable, même si d'autres facteurs (potentiel génétique — traitement sylvicole) ont une importance peut-être plus grande (PoLgE et alt. 1972).

Toutes ces questions d'interrelations sol hêtre passent par l'intermédiaire des problèmes de nutrition minérale. Or la nutrition minérale du hêtre n'a guère été étudiée.

D'une manière générale, d'ailleurs, les feuillus n'ont fait l'objet que de peu d'études de nutrition minérale, en raison de la priorité accordée aux reboisements résineux. Le peuplier fait toutefois exception à la règle, et le travail de Leroy (1968) sur le chêne a permis de combler une lacune.

Il était donc nécessaire de déterminer la variabilité des teneurs en éléments minéraux des feuilles de hêtre, au cours de l'année, afin de choisir la meilleure époque de prélèvement et la variabilité de ces mêmes éléments minéraux en fonction du type de station. En dehors de l'établissement de critères pour les diagnostics de nutrition, la connaissance de ces variations est importante pour la compréhension de l'influence du hêtre sur l'évolution du sol.

Cette étude a d'abord débuté en 1968 et 1969 dans deux stations (Vosges et plateaux calcaires de l'Est de la France) par des prélèvements mensuels. Elle a ensuite été poursuivie à partir de 1969 sur une soixantaine de stations situées sur une gamme de sols très étendue allant du podzol à la rendzine, par des prélèvements à deux périodes de l'année, le premier en août (feuilles vertes de la cime de l'arbre) et le second en octobre à la chute des feuilles (feuilles fraîchement tombées). La date du mois d'août a été choisie en fonction des premiers résultats de l'année 1968 et celle d'octobre en raison de la facilité de récolte des échantillons. Le travail à but purement méthodologique (établissement de critères de diagnostic de nutrition) apporte un certain nombre de données concernant la variabilité des éléments majeurs chez le hêtre et de quelques éléments moins fréquemment étudiés comme le fer et le manganèse. De plus les résultats obtenus ne sont pas sans intérêt pour la compréhension du cycle des éléments minéraux et de l'interaction essence type de station.

\section{1. - MATÉriel ET MÉTHODES}

\section{1. - Caractéristiques des stations}

Les deux stations, où ont été effectués les prélèvements mensuels, ont des caractéristiques pédologiques très différentes. L'une est située en forêt de Haye à $350 \mathrm{~m}$ d'altitude près de Nancy au lieu dit « La crédence ». Le sol est une rendzine développée sur un calcaire colluvionné (grouine). Le peu- 
plement est âgé de 120 ans environ et a une croissance moyenne. L'autre est situé dans les Vosges près du col de Hantz dans le bois de la Costelle à $700 \mathrm{~m}$ d'altitude. Le sol est un sol ocre podzolique à mor situé sur dévonodinantien. C'est un sol extrêmement désaturé très pauvre en calcium $(0,01 \mathrm{~m}$.e. $\mathrm{de} \mathrm{Ca}^{++}$pour $100 \mathrm{~g}$ de sol dans les horizons moyens).

Le peuplement est également âgé de plus de 100 ans et a une croissance très médiocre.

Les 65 autres peuplements choisis pour l'étude de la variabilité stationnelle se répartissent ainsi : 8 sont situés dans les Basses Vosges sur limon et grès, 15 sur des affleurements rhétiens (limons, grès, argiles), 36 sur des affleurements calcaires dont 8 sur sols entièrement carbonatés et 30 sur des sols plus ou moins complètement décarbonatés, et enfin 13 sont situés en Argonne dont 6 sur la gaize siliceuse et 7 sur des limons ou des limons sableux. Les types d'humus de ces différentes stations sont évidemment très variés depuis le mor jusqu'au mull calcique, en passant par tous les intermédiaires moder, mull-moder, mull-acide, mull-mésotrophe et mull-eutrophe, à l'exclusion des types d'humus hydromorphes.

\section{2. - Méthodes de prélèvements}

Les prélèvements mensuels ont été effectués, pour chacune des deux stations, sur trois arbres différents et à trois hauteurs différentes (tiers supérieur $\mathrm{H}$, tiers moyen $\mathrm{M}$, tiers inférieur B). Chaque échantillon comprenait des feuilles prélevées dans 8 directions différentes pour éliminer l'effet éventuel de l'orientation.

Les prélèvements pour l'étude de la variation stationnelle ont été effectués sur 5 arbres dominants par station entre le 15 et le 31 août 1969, dans le tiers supérieur de la cime. Les 5 échantillons ont ensuite été analysés séparément. La deuxième série de prélèvement a été effectuée à la fin du mois d'octobre 1969 par ramassage des feuilles tombées à terre, juste au début de la chute, qui commence par la cime de l'arbre. Un seul échantillon a donc été analysé par station pour cette deuxième série de prélèvements.

\section{3. - Méthodes d'analyses}

Tous les échantillons ont été séchés à $65^{\circ}$ à l'étuve puis broyés.

Le carbone a été dosé par pyrolyse oxydante au carmograph Wösthoff. L'azote a été dosé par distillation au micro-Kjeldähl Parnas-Wagner. Les autres éléments ont été dosés soit à partir d'une fusion alcaline des cendres obtenues par passage progressif de la poudre de feuilles jusqu'à $700^{\circ}$ au four à moufle, soit après attaque par voie humide (attaque perchlorique et à l'eau oxygénée). Le potassium, le calcium, le magnésium, le manganèse, le silicium et le fer ont été dosés soit par spectrophotométrie d'absorption, soit par spectrophotométrie d'émission. Le phosphore a été déterminé par
colorimétrie vanadomolybdique.

Les résultats sont exprimés en $\%$ ou en $\%$ de la matière sèche à $65^{\circ} \mathrm{C}$. La teneur en eau des feuilles est exprimée en \% par rapport à la matière sèche.

\section{4. - Méthodes d'interprétation statistique}

L'influence de la date de prélèvement et de la position d'échantillonnage a été testée par une analyse classique de la variance à 3 facteurs contrôlés (position, date et arbre).

$$
\begin{array}{lll}
3 \text { facteurs } & \text { No } 1 \text { position } 3 \text { niveaux (A) } \\
& \text { No } 2 \text { date } & 6 \text { niveaux (B) } \\
& \text { No } 3 \text { arbre } & 3 \text { niveaux (C) }
\end{array}
$$

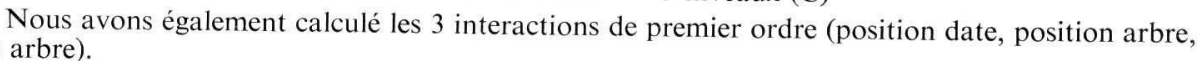
date arbre).

Nous avons ensuite calculé pour les points moyens (moyenne des 3 positions et des 3 arbres), les courbes de variation en fonction du temps, par régression polynomiale.

La variabilité stationnelle a été déterminée par analyse de la variance à un facteur contrôlé pour les prélèvements d'août et pour les deux dates par calcul de l'écart type de chaque élément.

Nous avons ensuite comparé la matrice des coefficients de corrélation entre éléments pour chacune des deux dates. Enfin, la reproductibilité du diagnostic entre août et octobre a été étudiée par calcul des coefficients d'intercorrélation entre éléments. 


\section{II. - RÉSULTATS}

\section{1. - Variation saisonnière}

En 1968 les prélèvements n'ont débuté que le 4 juillet et ont pris fin le 31 octobre. En 1969 , ils ont débuté au moment du débourrement (13 mai), et ont pris fin le 28 octobre à la chute des feuilles. Les résultats des deux années étant très comparables, nous commenterons les résultats plus complets de la seule année 1969.

\subsection{1. Étude des courbes de variation annuelle}

Carbone - azote - rapport C/N (fig. 1, 2 et 3 ).

La répartition du carbone dans les feuilles présente une grande analogie entre les deux stations : les feuilles de la station de la Costelle ont néanmoins une teneur en carbone plus forte que celles de la forêt de Haye. La teneur en carbone des feuilles commence par décrô̂tre du débourrement jusqu'au mois de juin; elle croît ensuite jusqu'en juillet, puis décroît à nouveau. Par régression polynomiale même jusqu'au troisième degré il n'est pas possible d'obtenir un ajustement significatif.

La teneur en azote des feuilles ne varie guère d'une station à l'autre. Dans le temps elle présente un maximum au débourrement ( $3,5 \%$ environ). Il existe un palier net entre le premier juillet et le premier septembre. Puis on observe à nouveau une décroissance rapide jusqu'à la chute des feuilles. Par régression polynomiale il est possible d'ajuster une courbe du $3^{\mathrm{e}}$ degré.

En ce qui concerne le rapport $\mathrm{C} / \mathrm{N}$, les différences entre les deux stations sont encore assez faibles tout au moins jusqu'au début septembre. Il existe un palier assez net au milieu de la période de végétation; la courbe de variation du rapport $\mathrm{C} / \mathrm{N}$ peut également être assimilée à une fonction du $3^{\mathrm{e}}$ degré.

Potassium - calcium - magnésium - (fig. 4, 5 et 6).

La variation de la teneur en potassium de feuilles peut aussi être représentée par une cubique. Il existe une relative stabilité en juillet et aô̂t, mais le palier est beaucoup moins net que pour l'azote ou le rapport $\mathrm{C} / \mathrm{N}$.

La teneur en calcium augmente continuellement dans les feuilles, depuis le débourrement jusqu'à leur chute. Les différences sont importantes entre les stations et s'accentuent en fonction du temps. Ces variations peuvent être représentées par une droite, dont le coefficient angulaire est différent pour les deux stations.

La variation de la teneur en magnésium est inverse de celle du calcium (maximum au débourrement). Elle peut également être représentée par une droite à coefficient angulaire différent pour les deux stations.

Manganèse - fer - silicium (fig. 7, 8 et 9).

Alors que la teneur en manganèse des feuilles du peuplement situé sur rendzine augmente au cours de l'année de 0,61 à $1,22 \%$ celle du peuplement situé sur sol ocre cryptopodzolique reste sensiblement constante $(0,85$ à $0,90 \%)$ sauf en fin de période de végétation où l'on observe une légère concentration $(1 \%)$. 
La teneur en fer varie relativement peu au cours de l'année. Elle augmente légèrement à l'automne et semble présenter un certain palier en août.

La teneur en silicium augmente très rapidement au cours du temps. Dans la station de Haye, la concentration initiale est de l'ordre de $0,80 \%$ et atteint $4,7 \%$ à la chute des feuilles. Dans la station de la Costelle les teneurs en silicium vont de $1,10 \%$ initialement à $6,5 \%$ en fin de période de végétation.

Phosphore (fig. 10).

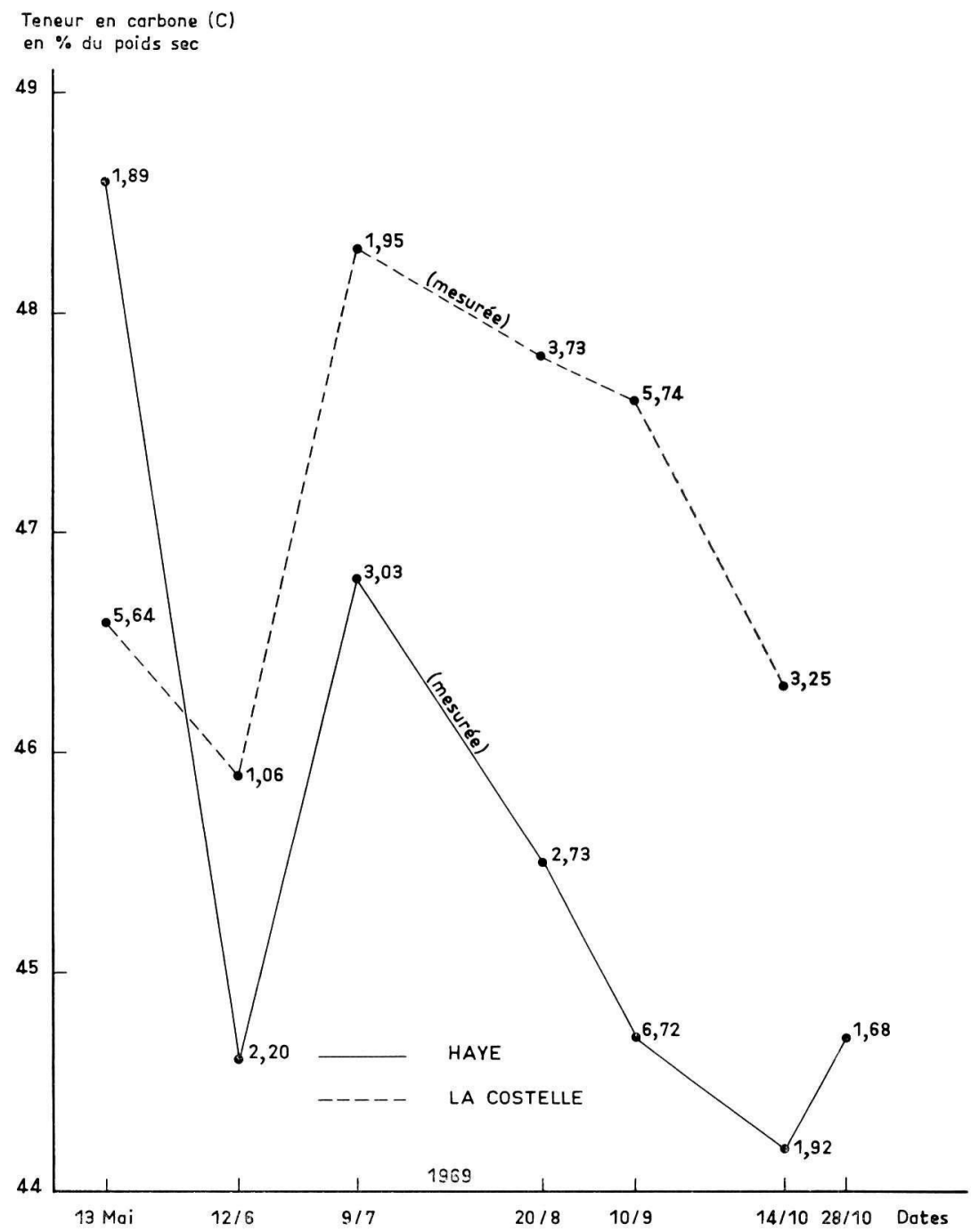

FIG. 1. - Variation de la teneur en carbone des feuilles de hêtre au cours de l'année 1969 (le chiffre porté en chaque point des courbes représente l'intervalle de confiance)

FIG. 1. - Variation of carbon content in beech leaves during year 1969 (in each point of the curves are indicated confidence intervals) 
La variation de la teneur en phosphore au cours de l'année peut être représentée par une cubique très semblable pour les deux stations. La teneur en phosphore est très forte au moment du débourrement (environ $0,43 \%$ en $\mathrm{P}$ ) puis décroît rapidement et se maintient autour de $0,15 \%$ pour les deux stations, du 15 juin au 15 septembre.

Poids sec et teneur en eau des feuilles (fig. 11 et 12).

Dans les deux stations la variation du poids de 100 feuilles en fonction du temps peut être représentée par une parabole. Le poids atteint un maximum vers le milieu du mois d'août.

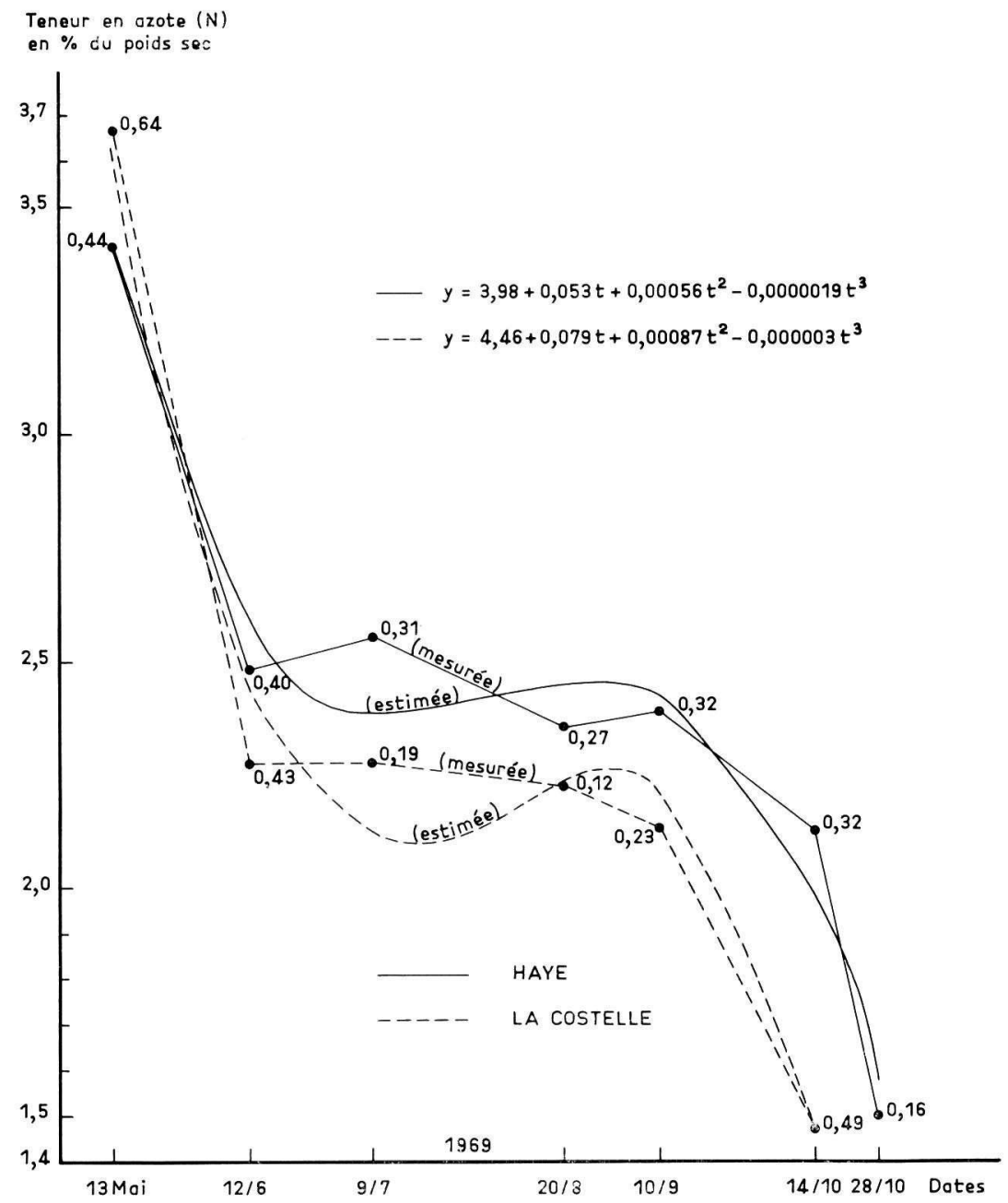

FIG. 2. - Variation de la teneur en azote des feuilles de hêtre au cours de l'année 1969 (le chiffre porté en chaque point des courbes représente l'intervalle de confiance)

FIG. 2. - Variation of nitrogen content in beech leaves during year 1969 (in each point of the curves are indicated confidence intervals) 
La teneur moyenne en eau des feuilles par rapport au poids sec est très élevée au débourrement (de l'ordre de 400 parties d'eau pour 100 parties de matière sèche). Elle décroît ensuite très rapidement et se maintient de juillet à octobre autour de $160 \%$, pour la station de Haye et $130 \%$ pour celle de la Costelle.

En résumé, pour la plupart des éléments, l'allure des courbes de variation en fonction du temps est très semblable dans les deux stations. Cependant la saison de végétation se termi-

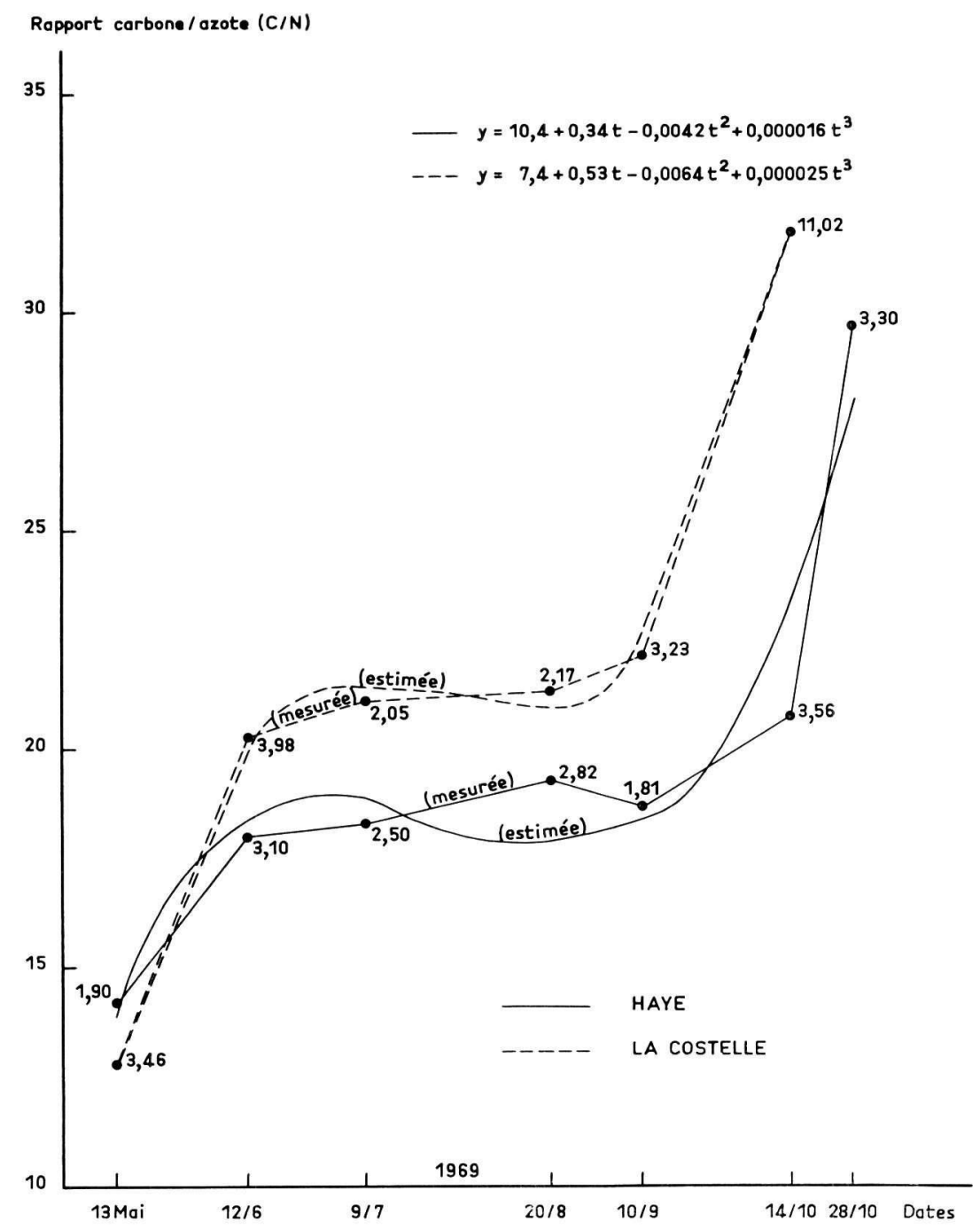

FIG. 3. - Variation du rapport $C / N$ des feuilles de hêtre au cours de l'année 1969 (le chiffre porté en chaque point des courbes représente l'intervalle de confiance)

FIG. 3. - Variation of $C / N$ ratio in beech leaves during year 1969 (in each point of the curves are indicated confidence intervals) 
nant à la Costelle 15 jours plus tôt qu'en forêt de Haye, l'allure des courbes, dont beaucoup sont assimilables à des cubiques, s'en ressent très légèrement.

Il apparaît que la teneur en éléments minéraux des feuilles du Bois de la Costelle est toujours inférieure à celle de la forêt de Haye, sauf pour le carbone et le silicium. D'autre part, si l'on excepte le calcium, pour lequel nous observons entre les deux stations un rapport de 1 à 2 , les écarts interstationnels sont toujours faibles (tableau $n^{\circ} 1$ ).

Enfin, nous constatons que les feuilles arrivent au sol au moment où les teneurs en calcium, manganèse, silicium et fer sont à leur niveau le plus élevé. Ce phénomène n'est pas sans influence sur le cycle biogéochimique de ces éléments comme l'ont montré plusieurs auteurs (Vedy et Duchaufour, 1971; Toutain et Duchaufour, 1970).

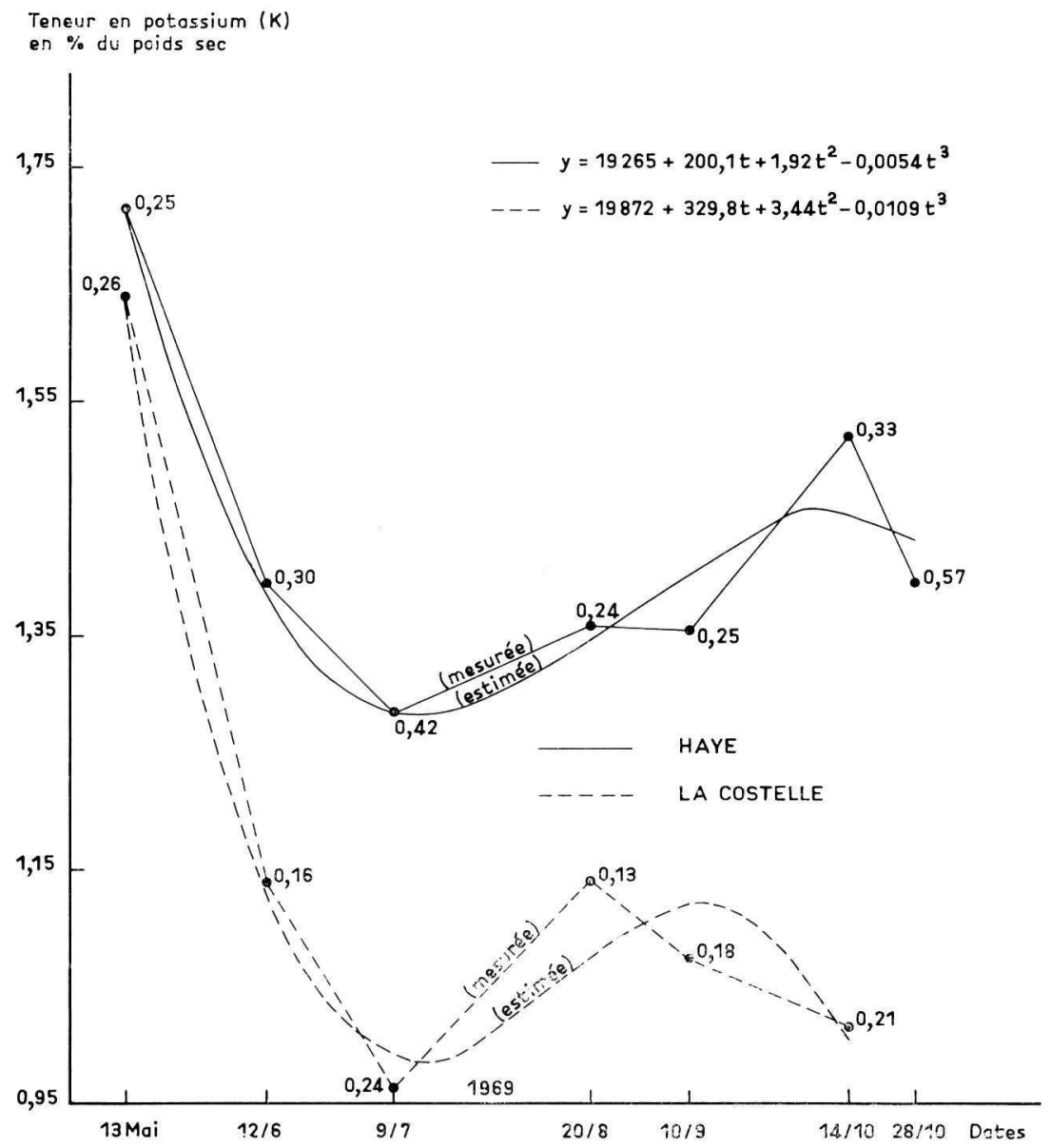

FIG. 4. - Variation de la teneur en potassium des feuilles de hêtre au cours de l'année 1969 (le chiffre porté en chaque point des courbes représente l'intervalle de confiance)

FIG. 4. - Variation of potassium content in beech leaves during year 1969 (in each point of the curves are indicated confidence intervals) 


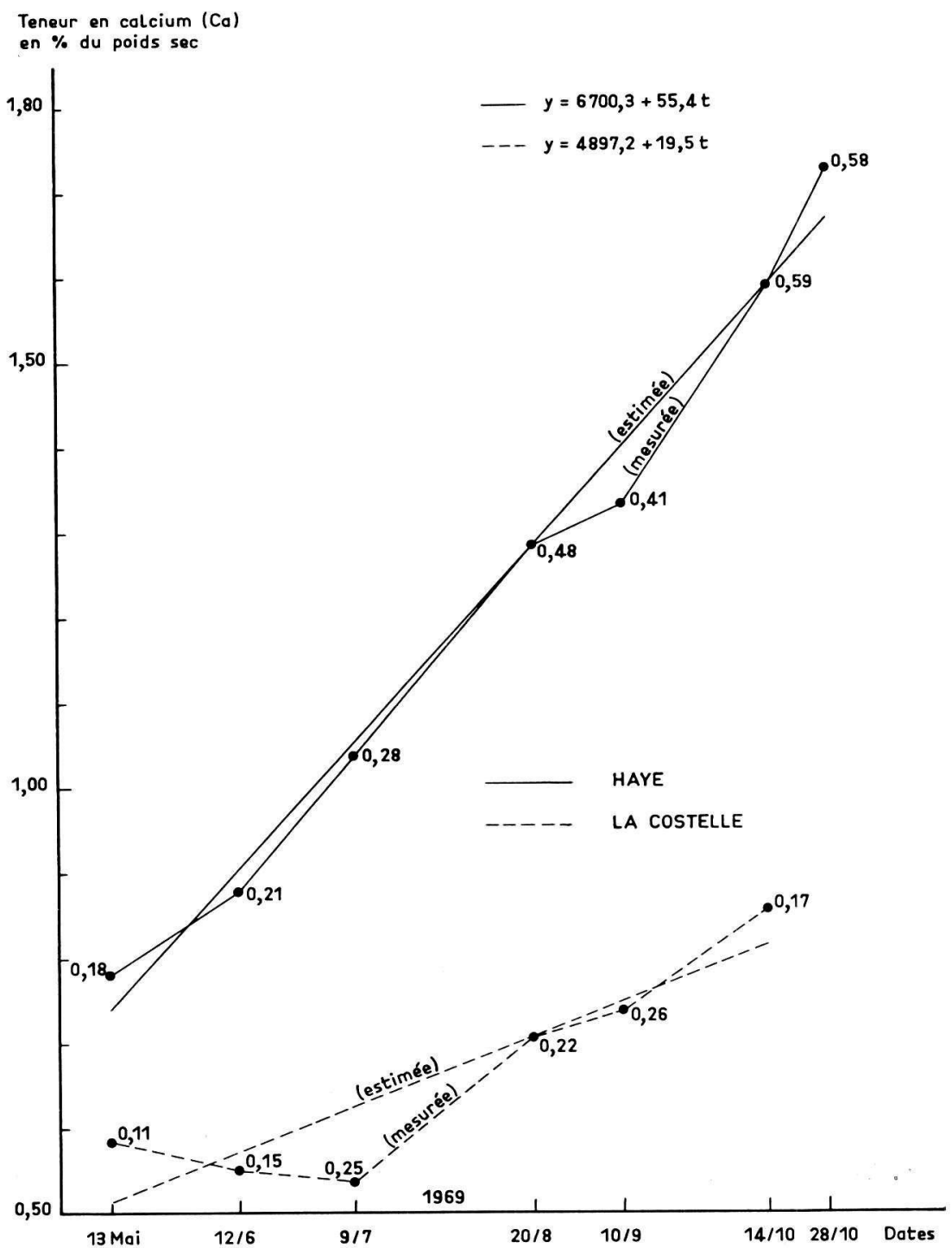

FIG. 5. - Variation de la teneur en calcium des feuilles de hêtre au cours de l'année 1969 (le chiffre porté en chaque point des courbes représente l'intervalle de confiance)

FIG. 5. - Variation of calcium content in beech leaves during year 1969 (in each point of the curves are indicated confidence intervals) 


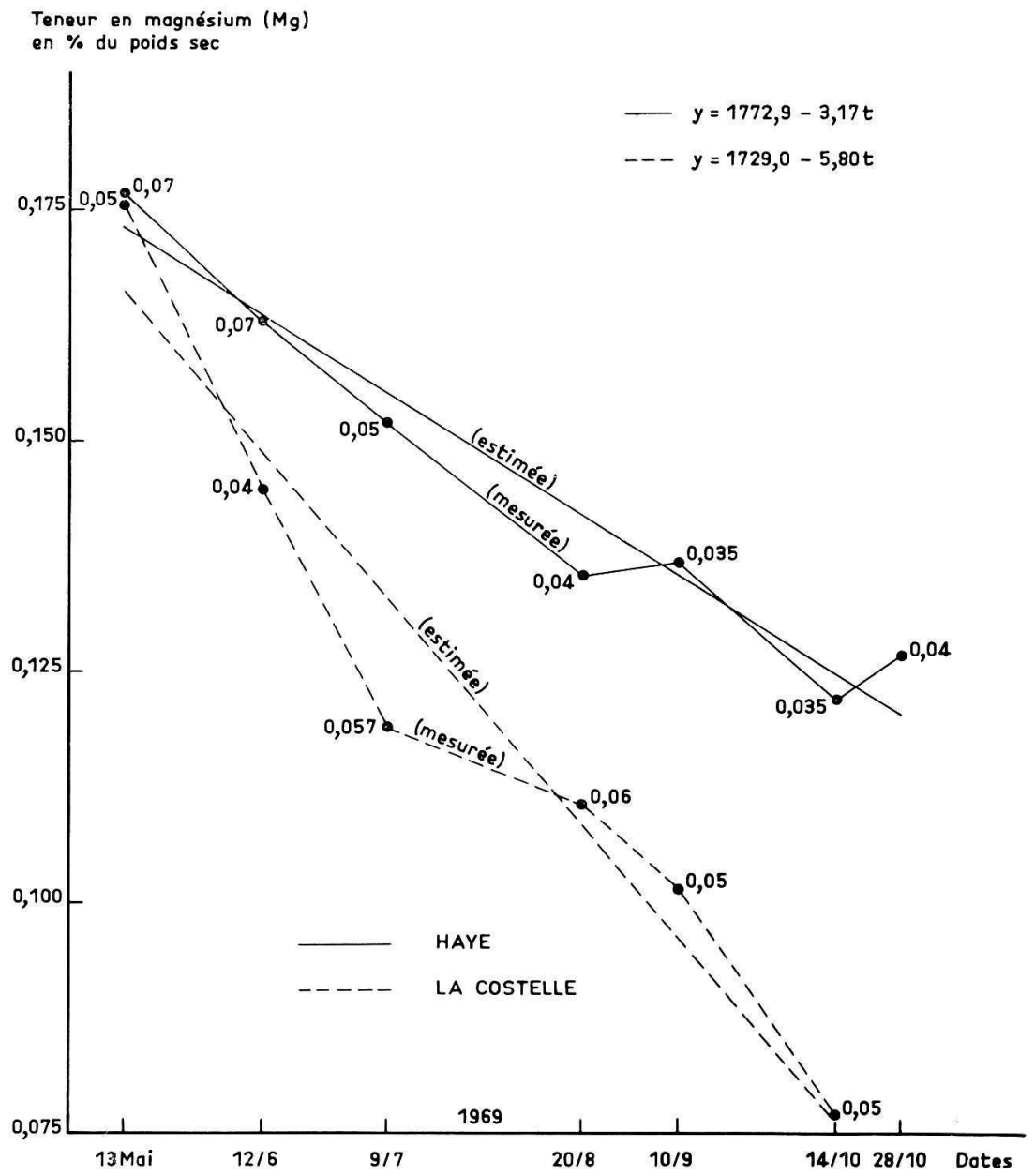

Fig. 6. - Variation de la teneur en magnésium des feuilles de hêtre au cours de l'année 1969 (le chiffre porté en chaque point des courbes représente l'intervalle de confiance)

FIG. 6. - Variation of magnesium content in beech leaves during year 1969 (in each point of the curves are indicated confidence intervals) 


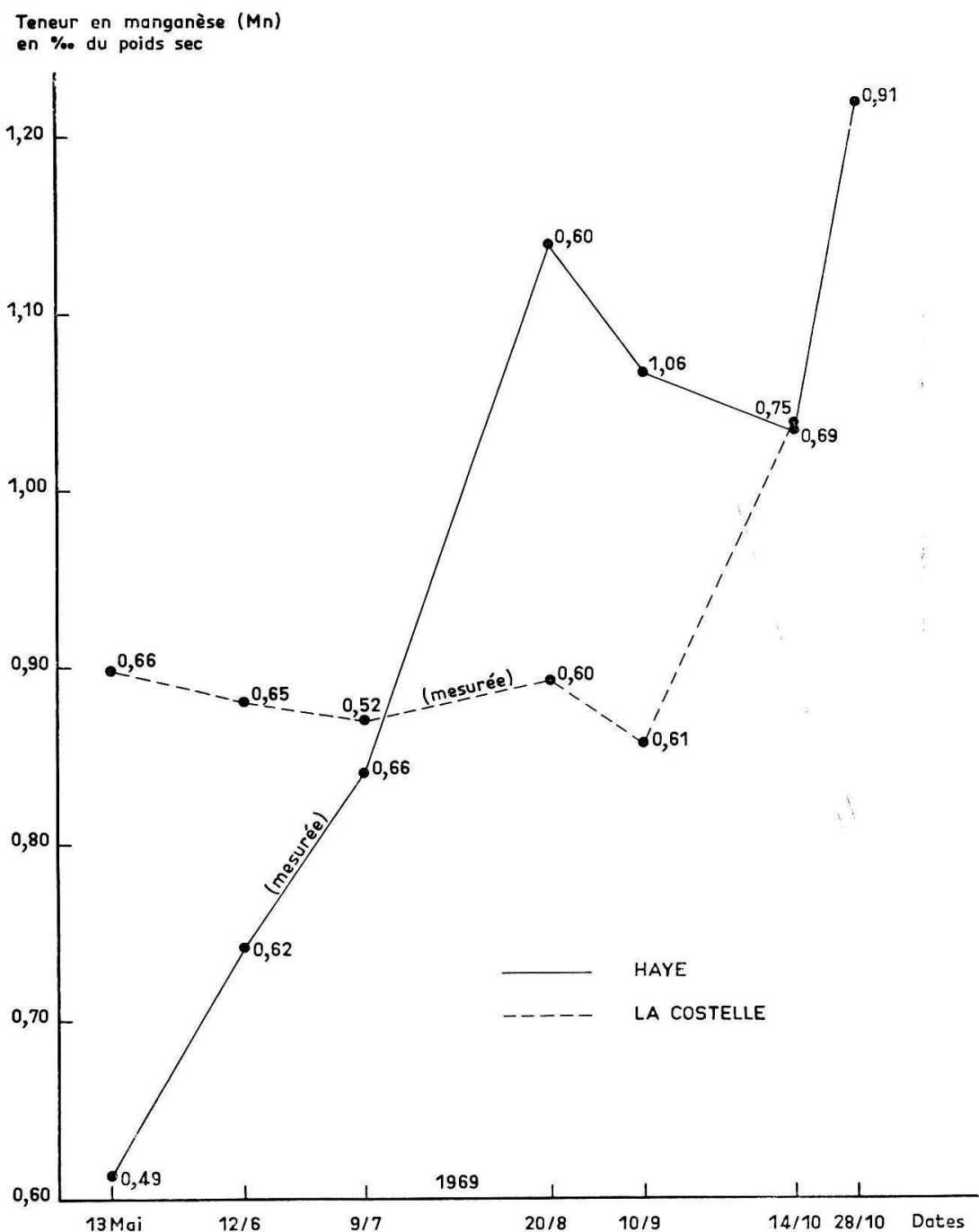

FIG. 7. - Variation de la teneur en manganèse des feuilles de hêtre au cours de l'année 1959 (le chiffre porté en chaque point des courbes représente l'intervalle de confiance)

FIG. 7. - Variation of manganese content in beech leaves during year 1969 (in each point of the curves are indicated confidence intervals) 


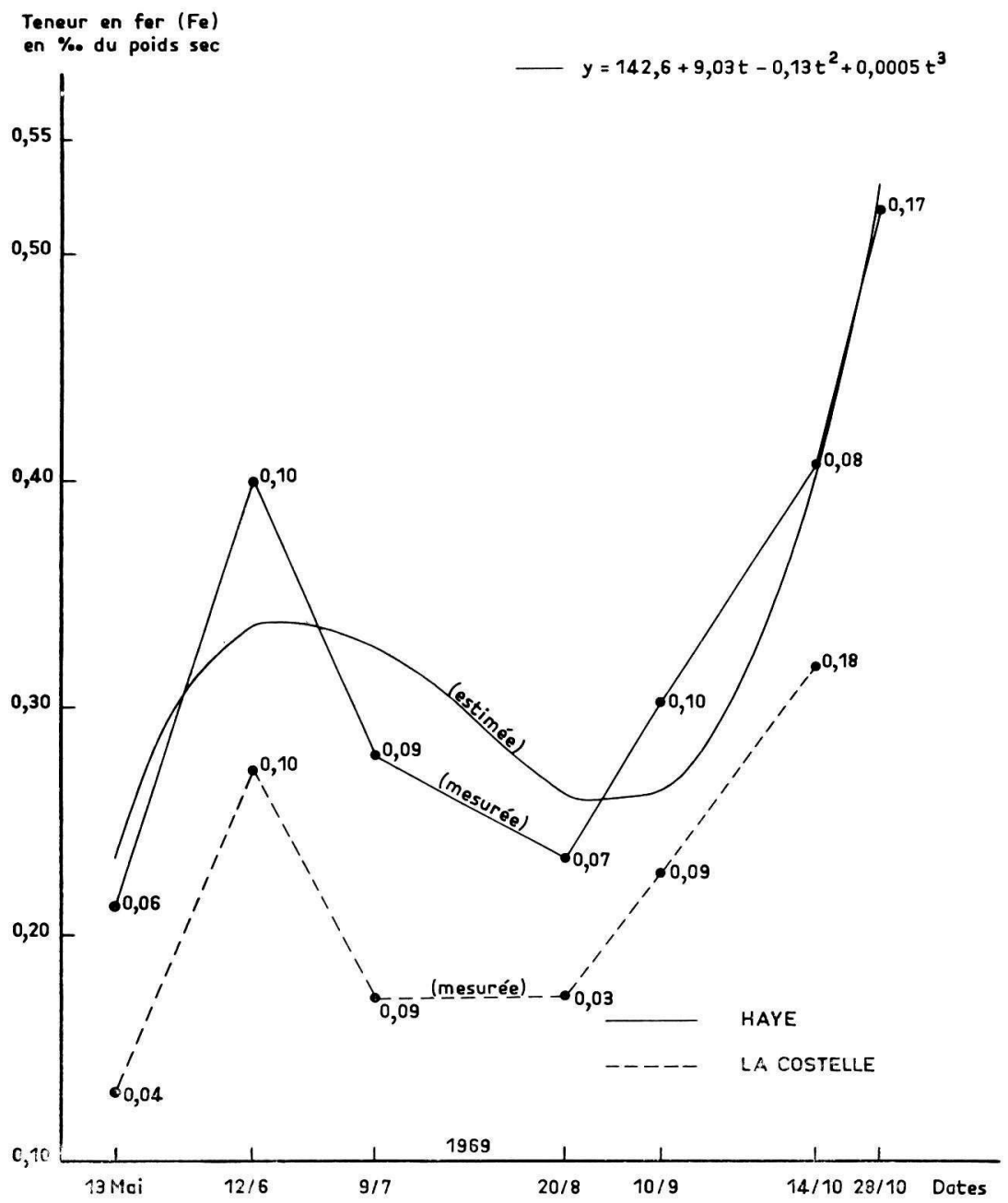

FIG. 8. - Variation de la teneur en fer des feuilles de hêtre au cours de l'année 1969 (le chiffre porté en chaque point des courbes représente l'intervalle de confiance)

FIG. 8. - Variation of iron content in beech leaves during year 1969 (in each point of the curves are indicated confidence intervals) 


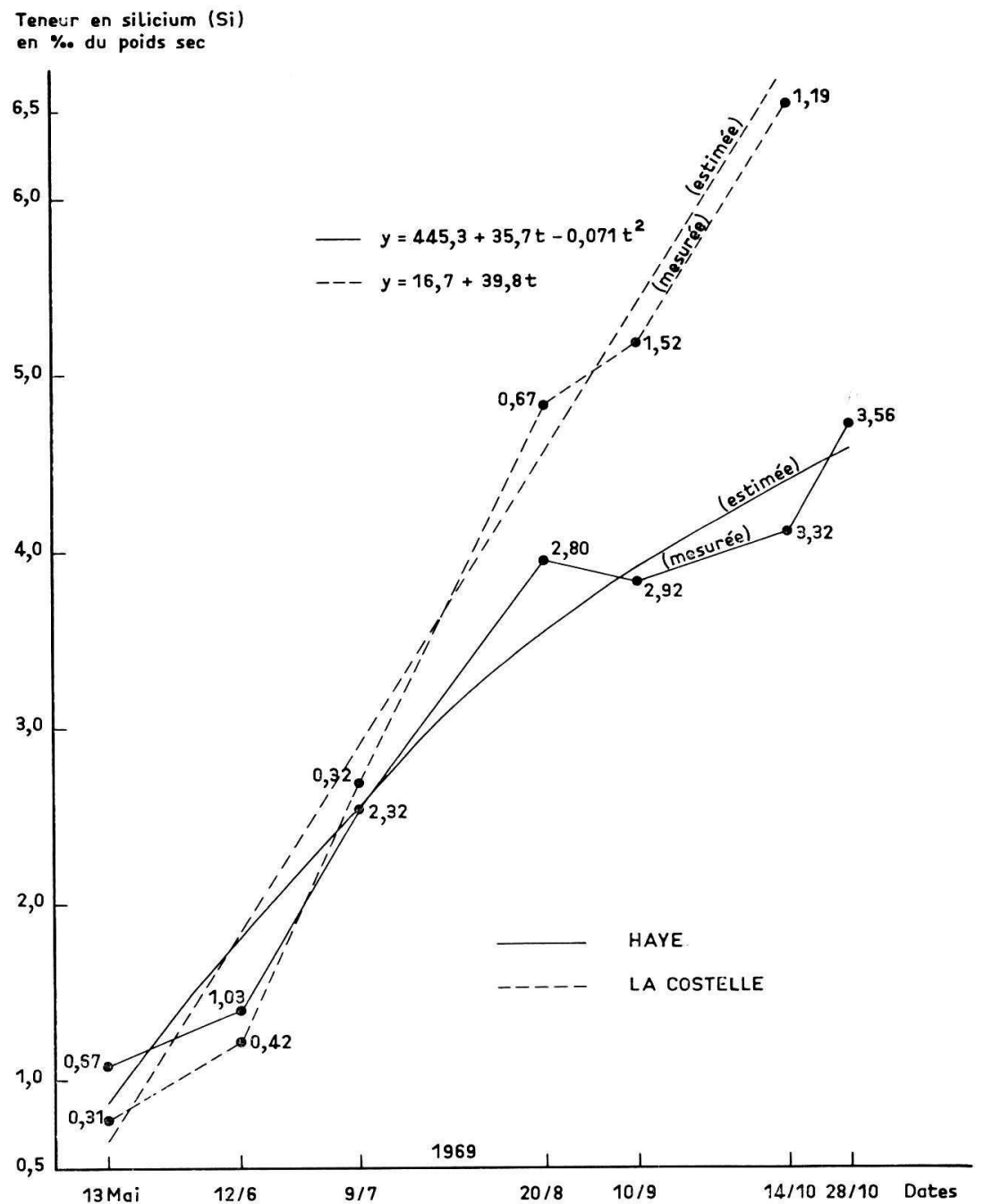

FIG. 9. - Variation de la teneur en silicium des feuilles de hêtre au cours de l'année 1969 (le chiffre porté en chaque point des courbes représente l'intervalle de confiance)

FIG. 9. - Variation of silicium content in beech leaves during year 1969 (in each point of the curves are indicated confidence intervals) 


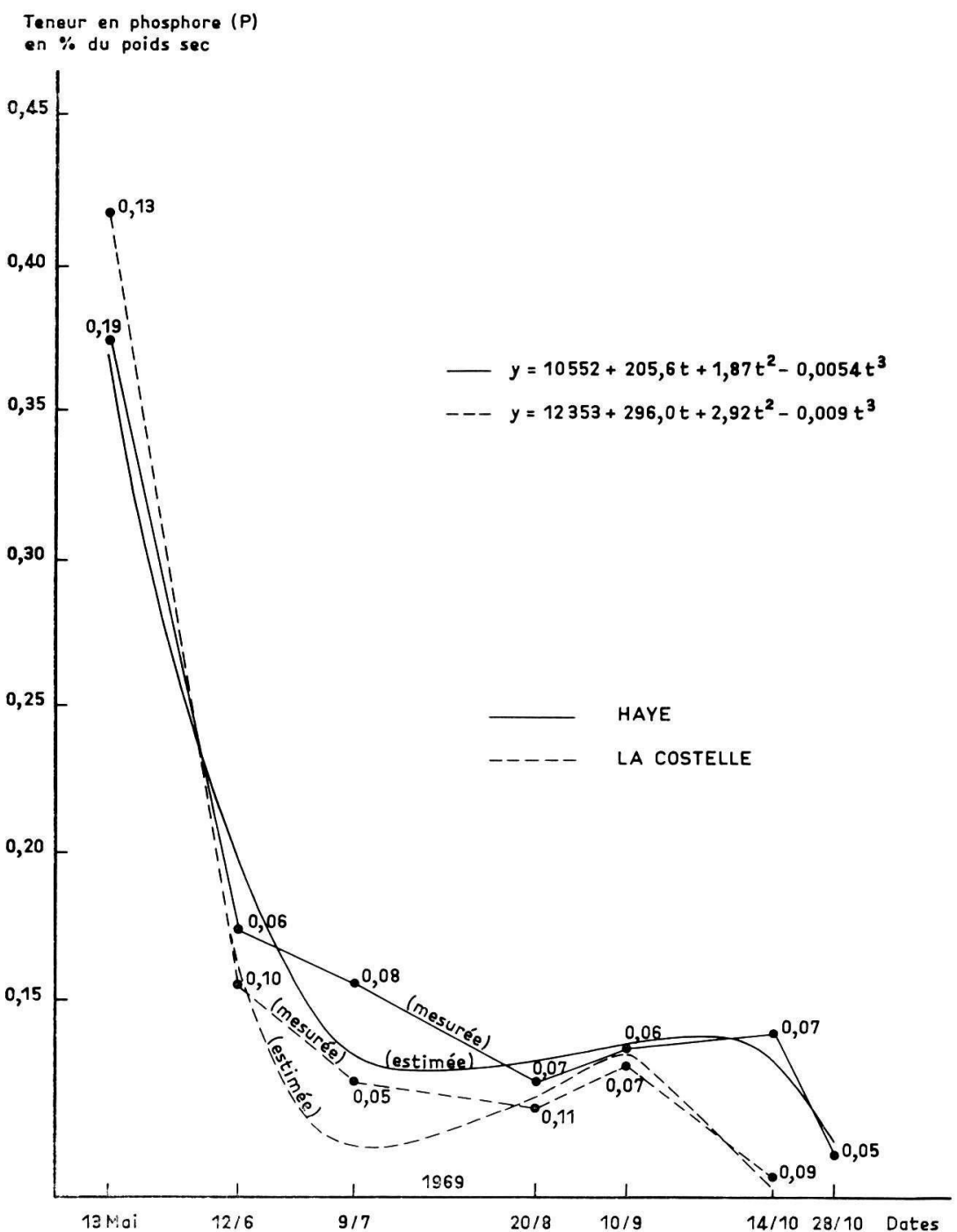

FIG. 10. - Variation de la teneur en phosphore des feuilles de hêtre au cours de l'année 1969 (le chiffre porté en chaque point des courbes représente l'intervalle de confiance)

FIG. 10. - Variation of phophorus content in beech leaves during year 1969 (in each point of the curves are indicated confidence intervals) 


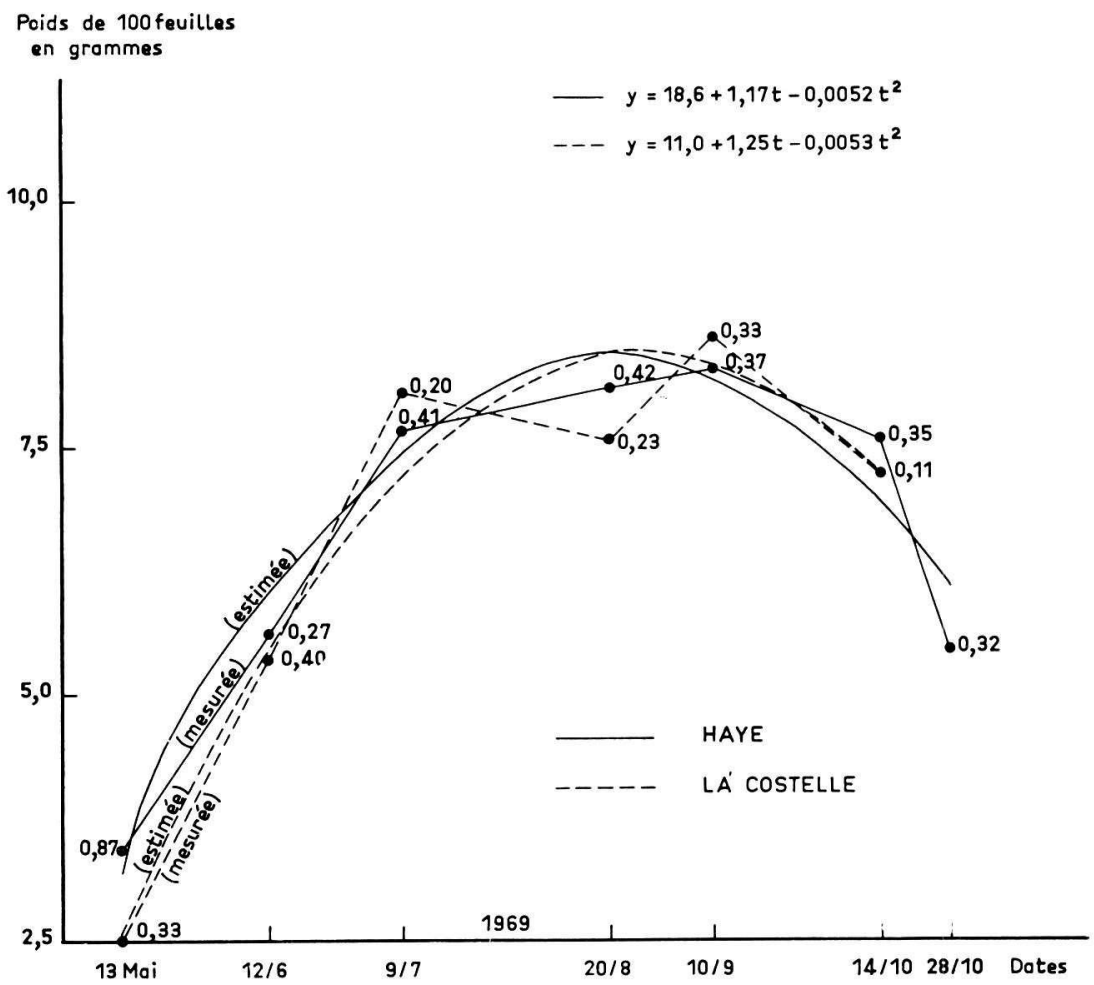

FIG. 11. - Variation du poids sec des feuilles de hêtre au cours de l'année 1969 (le chiffre porté en chaque point des courbes représente l'intervalle de confiance)

FIG. 11. - Variation of dry weight of beech leaves during year 1969 (in each point of the curves are indicated confidence intervals) 


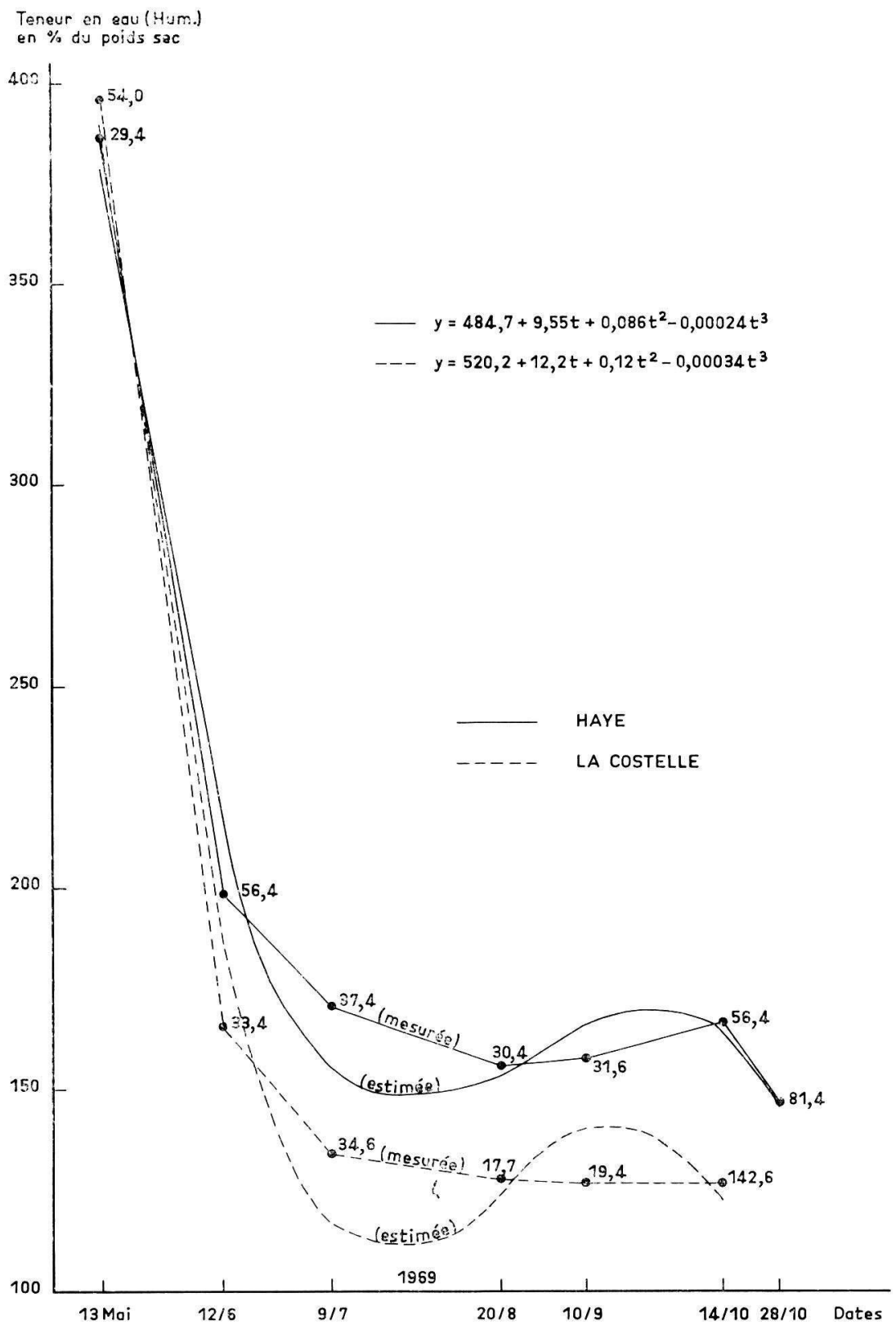

FIG. 12. - Variation de la teneur en eau (exprimée en \% par rapport au poids sec) des feuilles de hêtre au cours de l'année 1969

(le chiffre porté en chaque point des courbes représente l'intervalle de confiance)

FIG. 12. - Variation of water content (percentage of dry weight) of beech leaves during year 1969 (in each point of the curves are indicated confidence intervals) 


\section{TABLEAU 1 \\ TABLE 1}

Année 1959. Forêt de Haye. Analyse de la variance à 3 facteurs contrôlés : influence de la position d'échantillonnage, de la date de prélèvement de l'arbre, et de diverses interactions sur la composition foliaire des feuilles de hêtre.

1969 Haye Forest. Sample heigh, date, tree and interactions influence on mineral composition of beech leaves (variance analysis).

\begin{tabular}{|c|c|c|c|c|c|c|c|}
\hline \multirow{2}{*}{$\begin{array}{l}\text { Caractéristiques } \\
\text { des feuilles } \\
\text { de hêtre }\end{array}$} & \multicolumn{2}{|c|}{ Position } & \multirow{2}{*}{ Date } & \multirow{2}{*}{ Arbre } & \multicolumn{3}{|c|}{ Interactions } \\
\hline & $\begin{array}{l}\text { Signifi- } \\
\text { cation }\end{array}$ & $\begin{array}{c}\text { Teneur } \\
\text { moyenne }\end{array}$ & & & $\begin{array}{l}\text { Position } \\
\text { date }\end{array}$ & $\begin{array}{l}\text { Position } \\
\text { arbre }\end{array}$ & $\begin{array}{l}\text { Date } \\
\text { arbre }\end{array}$ \\
\hline $\mathrm{C} \%$ & & $\begin{array}{r}\text { H } 45,7 \\
\text { M } 45,5 \\
\text { B } 45,7\end{array}$ & $x \times$ & & & & \\
\hline $\mathrm{N} \%$ & $x \times$ & $\begin{array}{r}\text { H } 2,32 \\
\text { M } 2,44 \\
\text { B } 2,48\end{array}$ & $x \times$ & $x$ & & & \\
\hline $\mathrm{C} / \mathrm{N}$ & $x$ & $\begin{array}{cc}\text { H } & 20,6 \\
\text { M } & 19,6 \\
\text { B } & 19,3\end{array}$ & $x \times$ & & & & \\
\hline $\mathrm{K} \%$ & $x$ & $\begin{aligned} \text { H } & 1,40 \\
\text { M } & 1,40 \\
\text { B } & 1,49\end{aligned}$ & $x \times$ & $x \times$ & $\times x$ & & $x \times$ \\
\hline $\mathrm{Ca} \%$ & $x$ & $\begin{array}{cc}\text { H } & 1,19 \\
\text { M } & 1,20 \\
\text { B } & 1,30\end{array}$ & $x \times$ & $x \times$ & & $x \times$ & $x \times$ \\
\hline $\mathrm{Mg} \%$ & $\times$ & $\begin{array}{c}\text { H } 0,13 \\
\text { M } 0,14 \\
\text { B } 0,17\end{array}$ & $\times \times$ & $x \times$ & & $x \times$ & \\
\hline $\mathrm{Mn} \%$ & $x \times$ & $\begin{array}{r}\text { H } 0,59 \\
\text { M } 0,91 \\
\text { В } 1,34\end{array}$ & $\times x$ & $\times \times$ & & $x$ & $\times x$ \\
\hline $\mathrm{Si} \%$ & & $\begin{array}{c}\text { H } 3,05 \\
\text { M } 3,14 \\
\text { B } 3,09\end{array}$ & $x \times$ & $x \times$ & & & $x \times$ \\
\hline $\mathrm{Fe} \%$ & $x \times$ & $\begin{array}{r}\text { H } 0,32 \\
\text { M } 0,32 \\
\text { B } 0,36\end{array}$ & $x \times$ & $x \times$ & & $x$ & $x$ \\
\hline P \% & & $\begin{array}{r}\text { H } 0,19 \\
\text { M } 0,19 \\
\text { B } 0,19\end{array}$ & $x \times$ & $x \times$ & & & $x \times$ \\
\hline Eau \% & & $\begin{array}{c}\text { H } 193 \\
\text { M } 201 \\
\text { B } 200\end{array}$ & $x \times$ & $x \times$ & & & \\
\hline $\begin{array}{l}\text { Poids de } \\
100 \text { feuilles }\end{array}$ & $x \times$ & $\begin{array}{r}\text { H } 7,67 \\
\text { M } 6,65 \\
\text { B } 5,37\end{array}$ & $\times x$ & & & $x \times$ & $x$ \\
\hline
\end{tabular}




\section{TABLEAU 2}

\section{TABLE 2}

Année 1969. La Costelle. Analyse de la variance à 3 facteurs contrôlés : influence de la position d'échantillonnage de la date de prélèvement de l'arbre et de diverses interactions sur la composition des feuilles de hêtre.

1969 La Costelle Forest. Sample heigh, date, tree and interactions influence on mineral composition of beech leaves (variance analysis).

\begin{tabular}{|c|c|c|c|c|c|c|c|}
\hline \multirow{2}{*}{$\begin{array}{c}\text { Caractéristiques } \\
\text { des feuilles } \\
\text { de hêtre }\end{array}$} & \multicolumn{2}{|c|}{ Position } & \multirow{2}{*}{ Date } & \multirow{2}{*}{ Arbre } & \multicolumn{3}{|c|}{ Interactions } \\
\hline & $\begin{array}{l}\text { Signifi- } \\
\text { cation }\end{array}$ & $\begin{array}{l}\text { Teneur } \\
\text { moyenne }\end{array}$ & & & $\begin{array}{l}\text { Position } \\
\text { date }\end{array}$ & $\begin{array}{l}\text { Position } \\
\text { arbre }\end{array}$ & $\begin{array}{l}\text { Date } \\
\text { arbre }\end{array}$ \\
\hline $\mathrm{C} \%$ & & $\begin{array}{r}\text { H } 47,6 \\
\text { М } 46,8 \\
\text { В } 46,8\end{array}$ & & & & & \\
\hline $\mathrm{N} \%$ & & $\begin{array}{r}\text { H } 2,32 \\
\text { M } 2,35 \\
\text { B } 2,38\end{array}$ & $x \times$ & $x \times$ & x & $x$ & $x \times$ \\
\hline $\mathrm{C} / \mathrm{N}$ & $\times x$ & $\begin{array}{r}\text { H } 22,3 \\
\text { M } 21,1 \\
\text { B } 21,3\end{array}$ & $x \times$ & $x \times$ & & $x$ & $x \times$ \\
\hline $\mathrm{K} \%$ & & $\begin{array}{r}\mathrm{H} 1,15 \\
\mathrm{M}^{1} 1,18 \\
\mathrm{~B}^{1} 1,15\end{array}$ & $x \times$ & & & $x$ & \\
\hline $\mathrm{Ca} \%$ & $x \times$ & $\begin{array}{r}\text { H } 0,63 \\
\text { M } 0,64 \\
\text { В } 0,71\end{array}$ & $x \times$ & $x \times$ & & & \\
\hline $\mathrm{Mg} \%$ & $\times x$ & $\begin{array}{r}\text { H } 0,11 \\
\text { M } 0,11 \\
\text { B } 0,13\end{array}$ & $x \times$ & $x$ & & & \\
\hline $\mathrm{Mn} \%$ & x & $\begin{array}{r}\text { H } 0,85 \\
\text { M } 0,89 \\
\text { В } 0,97\end{array}$ & & $x \times$ & & & $x$ \\
\hline $\mathrm{Si} \%$ & & $\begin{array}{r}\text { H } 3,48 \\
\text { M } 3,60 \\
\text { B } 3,55\end{array}$ & $x \times$ & $x$ & & & $\times x$ \\
\hline $\mathrm{Fe} \%$ & & $\begin{array}{r}\text { H } 0,20 \\
\text { M } 0,21 \\
\text { B } 0,23\end{array}$ & $x \times$ & & & & \\
\hline $\mathrm{P} \%$ & $x \times$ & $\begin{array}{rr}\text { H } & 0,18 \\
\text { M } & 0,19 \\
\text { B } & 0,19\end{array}$ & $x \times$ & $x$ & & & $x \times$ \\
\hline Eau \% & & $\begin{array}{rr}\text { H } & 182 \\
\text { M } & 180 \\
\text { B } & 177\end{array}$ & $\times x$ & & & & \\
\hline $\begin{array}{c}\text { Poids sec } \\
\text { de } 100 \text { feuilles }\end{array}$ & $x \times$ & $\begin{array}{r}\text { H } 7,87 \\
\text { M } 6,54 \\
\text { B } 5,11\end{array}$ & $x \times$ & $x \times$ & & $x \times$ & \\
\hline
\end{tabular}




\subsection{Influence de la hauteur des prélèvements et interaction hauteur - date - arbre}

Cinq caractères seulement présentent dans les deux stations des différences significatives suivant la hauteur de prélèvement (tableau 1 et 2 ). Il s'agit du rapport $\mathrm{C} / \mathrm{N}$, du calcium, du magnésium, du manganèse et du poids de 100 feuilles. Il n'existe jamais d'interaction dateposition pour ces cinq caractères. Leurs courbes de variation annuelle, corrigées en fonction de la hauteur de prélèvement, peuvent donc être facilement obtenues. Il suffit de tracer des courbes parallèles aux couches moyennes, en les décalant suivant les valeurs indiquées aux tableaux 1 et 2 .

Le rapport $\mathrm{C} / \mathrm{N}$ est dans les deux stations un peu plus élevé dans le haut de la cime que dans le bas. Il en est de même pour le poids des feuilles, les feuilles de lumière étant plus lourdes que les feuilles d'ombre. Au contraire, les teneurs en calcium et en magnésium sont un peu plus élevées dans les feuilles d'ombre que dans les feuilles de lumière. La teneur en manganèse est beaucoup plus forte dans les feuilles d'ombre que dans les feuilles de lumière (forêt de Haye $1,36 \%$ et $0,59 \%$ ).

Les autres caractères présentent des différences significatives suivant la position d'échantillonnage, mais soit dans une station, soit dans l'autre. Il s'agit du potassium (Haye), de l'azote (Haye), du fer (Haye) et du phosphore (La Costelle). Quand les différences entre les prélèvements du haut et ceux du bas de la cime sont significatives, on remarque que les feuilles d'ombre sont toujours plus riches que les feuilles de lumière.

Enfin, 3 caractères ne présentent pas de différence significative entre les feuilles d'ombre et de lumière. Il s'agit de la teneur en eau, de la teneur en carbone, et de la teneur en silicium.

L'analyse de la variance à 3 facteurs contrôlés montre qu'il existe un grand nombre d'interactions significatives entre les variations des teneurs en différents éléments minéraux suivant la date de prélèvement, la position et l'arbre échantillonné.

Les interactions arbre-date, et arbre-position sont assez fréquentes ce qui montre que chaque arbre a un comportement particulier et indépendant de la station où il se trouve. L'ensemble de ces interactions est résumé par les tableaux 1 et 2.

\section{2. - Variations stationnelles}

\subsection{Prélèvements effectués en août.}

\subsection{Jugement en valeur absolue.}

Nous ne connaissons guère pour le hêtre les niveaux optima ou les seuils de carence des divers éléments présents dans les feuilles. Il est donc difficile de situer les peuplements étudiés par rapport à des normes. Néanmoins, comme ces peuplements s'étagent des plus basses fertilités aux plus hautes, il est possible d'avoir une idée des teneurs optimales et des seuils de déficience en prenant, du moins pour les éléments principaux $\mathrm{N}, \mathrm{P}$ et $\mathrm{K}$ les valeurs maximales et les valeurs minimales observées (tableau 3).

Il serait imprudent de faire le même raisonnement pour d'autres éléments majeurs (Ca par exemple) qui peuvent s'accumuler par " consommation de luxe ", sans que la production en soit améliorée. Il en est de même pour les oligo-éléments ( $\mathrm{Mn}, \mathrm{Fe}$ ). Récipro- 
TABLEAU 3

TABLE 3

Variations stationnelles de la teneur en éléments minéraux des feuilles de hêtre. Prélèvements du mois d'août 1969 (Moyenne - écart type - maximum et minimum) (65 peuplements).

Site variation of mineral composition of beech leaves in August 1969; Averages, standard deviation, maximum, minimum (65 sites).

\begin{tabular}{c|c|c|c|c}
\hline \hline $\begin{array}{c}\text { Éléments minéraux } \\
\text { totaux des feuilles } \\
\text { de hêtre }\end{array}$ & $\begin{array}{c}\text { Moyenne } \\
\text { observée }\end{array}$ & Écart type & $\begin{array}{c}\text { Maximum } \\
\text { observé }\end{array}$ & $\begin{array}{c}\text { Minimum } \\
\text { observé }\end{array}$ \\
\hline $\mathrm{N} \%$ & 2,02 & 0,117 & 2,24 & 1,77 \\
$\mathrm{P} \%$ & 0,16 & 0,06 & 0,29 & 0,125 \\
$\mathrm{~K} \%$ & 0,89 & 0,136 & 1,23 & 0,63 \\
$\mathrm{Ca} \%$ & 1,21 & 0,347 & 1,83 & 0,63 \\
$\mathrm{Mg} \%$ & 0,16 & 0,03 & 0,25 & 0,07 \\
$\mathrm{Mn} \%$ & 1,45 & 1,28 & 4,71 & 0,22 \\
$\mathrm{Fe} \%$ & 0,16 & 0,09 & 0,39 & 0,04 \\
\hline
\end{tabular}

quement les concentrations les plus basses obtenues pour ces éléments n'ont pas forcément un effet limitatif.

En attendant les résultats des essais de fertilisation en cours, et de l'étude des liaisons entre croissance et éléments présents dans le sol ou les feuilles, ces valeurs minimales peuvent nous permettre d'établir une première approximation.

Nous pouvons donc considérer, mais avec prudence, que l'optimum de la teneur en azote des feuilles de hêtre dans la deuxième quinzaine d'août se situerait aux environs de $2,25 \%$, l'optimum de la teneur en phosphore (P) aux environs de $0,28 \%$ et l'optimum de la teneur en potassium aux environs de $1,20 \%$.

\subsection{Analyse de la variance à un facteur contrôlé.}

Les résultats de cette analyse sont donnés dans le tableau 4. Tous les tests $F$ sont significatifs. Globalement les peuplements étudiés différent donc par tous les éléments dosés. Si nous classons les éléments en fonction de la valeur de F, nous constatons que c'est le manganèse qui discrimine le plus l'ensemble des peuplements. Le manganèse est suivi du calcium, puis du fer et du phosphore. La faible discrimination de la nutrition azotée est assez surprenante. Nous reviendrons sur ce point dans la discussion. 
TABLEAU 4

TABLE 4

Prélèvements du mois d'août 1969. Analyse de la variance à un facteur contrôlé sur les éléments minéraux des feuilles de hêtre ( 5 prélèvements par peuplement) 65 peuplements.

Samples of August 1969. Variance analysis on mineral elements of beech leaves (65 sites and 5 simples in each site).

\begin{tabular}{c|c|c}
\hline \hline Caractère & $\begin{array}{c}\text { Test F } \\
\text { ddl }=64-258\end{array}$ & Signification \\
\hline $\mathrm{Mn}$ & 35,20 & $\times \times$ \\
$\mathrm{Ca}$ & 17,38 & $\times \times$ \\
$\mathrm{Fe}$ & 12,71 & $\times \times$ \\
$\mathrm{P}$ & 11,06 & $\times \times$ \\
$\mathrm{K}$ & 3,99 & $\times \times$ \\
$\mathrm{N}$ & 2,80 & $\times \times$ \\
$\mathrm{Mg}$ & 2,78 & $\times \times$ \\
\hline
\end{tabular}

2.213. Analyse de la matrice des coefficients de corrélation.

La matrice des coefficients de corrélation totaux (fig. 13) est intéressante à examiner. Le calcium est lié négativement à 4 éléments : le manganèse, le phosphore, l'azote et le potassium. La liaison calcium manganèse est particulièrement élevée puisqu'elle atteint $-0,75$. Toutes les autres liaisons observées sont positives et beaucoup moins fortes ( $\mathrm{N}-\mathrm{Fe}, \mathrm{N}-\mathrm{K}$ et $\mathrm{P}-\mathrm{Fe})$.

\subsection{Prélèvements effectués en octobre à la chute des feuilles.}

\subsection{Jugement en valeur absolue.}

Si les niveaux optima et les seuils de carence ne sont guère connus en pleine période de végétation, ils le sont encore moins en fin de période de végétation. Il est néanmoins intéressant d'examiner les résultats obtenus, dans le double optique d'un diagnostic de nutrition et d'une comparaison entre stations.

En moyenne la teneur en azote, phosphore et potassium a baissé dans les feuilles par rapport aux teneurs du mois d'août alors que la teneur en calcium, manganèse et fer a augmenté. Ces résultats sont conformes aux lois générales établies et aux courbes de variation décrites dans les paragraphes précédents.

Pour l'ensemble des 60 peuplements, la teneur en magnésium reste à peu près constante entre le mois d'août et la chute des feuilles.

Nous pouvons également noter que les écarts types ont tendance à augmenter d'août à octobre, pour tous les éléments, sauf pour le potassium. La variabilité entre peuplements est donc plus importante, en automne à la chute des feuilles, qu'en pleine périołe de végétation. 
TABLEAU 5

TABLE 5

Variations stationnelles de la teneur en éléments minéraux des feuilles de hêtre. Prélèvements d'octobre 1969. (Moyenne - écart type - maximum et minimum) (65 peuplements).

Site variations of mineral composition of beech leaves in October 1969. Average, standard deviation, maximum minimum ( 65 sites).

\begin{tabular}{c|c|c|c|c}
\hline \hline $\begin{array}{c}\text { Éléments minéraux } \\
\text { totaux des feuilles }\end{array}$ & $\begin{array}{c}\text { Moyenne } \\
\text { observée }\end{array}$ & Écart type & $\begin{array}{c}\text { Maximum } \\
\text { observé }\end{array}$ & $\begin{array}{c}\text { Minimum } \\
\text { observé }\end{array}$ \\
\cline { 2 - 5 } $\mathrm{N} \%$ & 0,923 & 0,148 & 1,34 & 0,63 \\
$\mathrm{P} \%$ & 0,09 & 0,08 & 0,19 & 0,05 \\
$\mathrm{~K} \%$ & 0,639 & 0,111 & 0,89 & 0,40 \\
$\mathrm{Ca} \%$ & 1,454 & 0,46 & 2,41 & 0,48 \\
$\mathrm{Mg} \%$ & 0,13 & 0,06 & 0,27 & 0,04 \\
$\mathrm{Mn} \%$ & 2,19 & 1,99 & 7,04 & 0,18 \\
$\mathrm{Fe} \%$ & 0,33 & 0,25 & 0,85 & 0,13 \\
\hline \hline
\end{tabular}

\subsection{Analyse de la matrice des coefficients de corrélation totaux.}

La matrice des coefficients de corrélation totaux présente beaucoup plus de liaisons significatives en automne qu'en été (fig. 13).

Nous retrouvons les liaisons négatives du calcium avec les mêmes éléments qu'en été : liaison $\mathrm{Ca}-\mathrm{Mn}$, Ca-P, Ca-N et Ca-K. Le coefficient de corrélation calcium manganèse est encore plus élevé en automne qu'en été, et atteint $-0,80$, ce qui est considérable. Nous retrouvons également les liaisons $\mathrm{N}-\mathrm{Fe}$ et $\mathrm{N}-\mathrm{K}$, avec en plus une liaison très significative N-P. La liaison Fe-P est également présente et renforcée. Enfin, un certain nombre de liaisons supplémentaires apparaissent : Mn-P, Mn-K, Mg-K, Mn-Fe.

Il est probable que ce sont les phénomènes de migrations des éléments minéraux vers les parties inférieures de l'arbre, qui sont la cause du renforcement des liaisons entre éléments.

\section{3. - Comparaison de la reproductibilité du diagnostic de nutrition entre les deux dates de prélèvement}

L'examen, aux deux dates de prélèvement, de la variation des écarts types et de la matrice des coefficients de corrélation entre éléments, montrent qu'il doit exister un certain nombre de distortions entre le diagnostic que l'on peut porter au mois d'août, et celui que l'on peut porter en automne.

Le calcul pour les différents éléments des coefficients d'intercorrélation entre les concentrations aux deux dates permet de préciser le sens de ces distortions (tableau 6). 

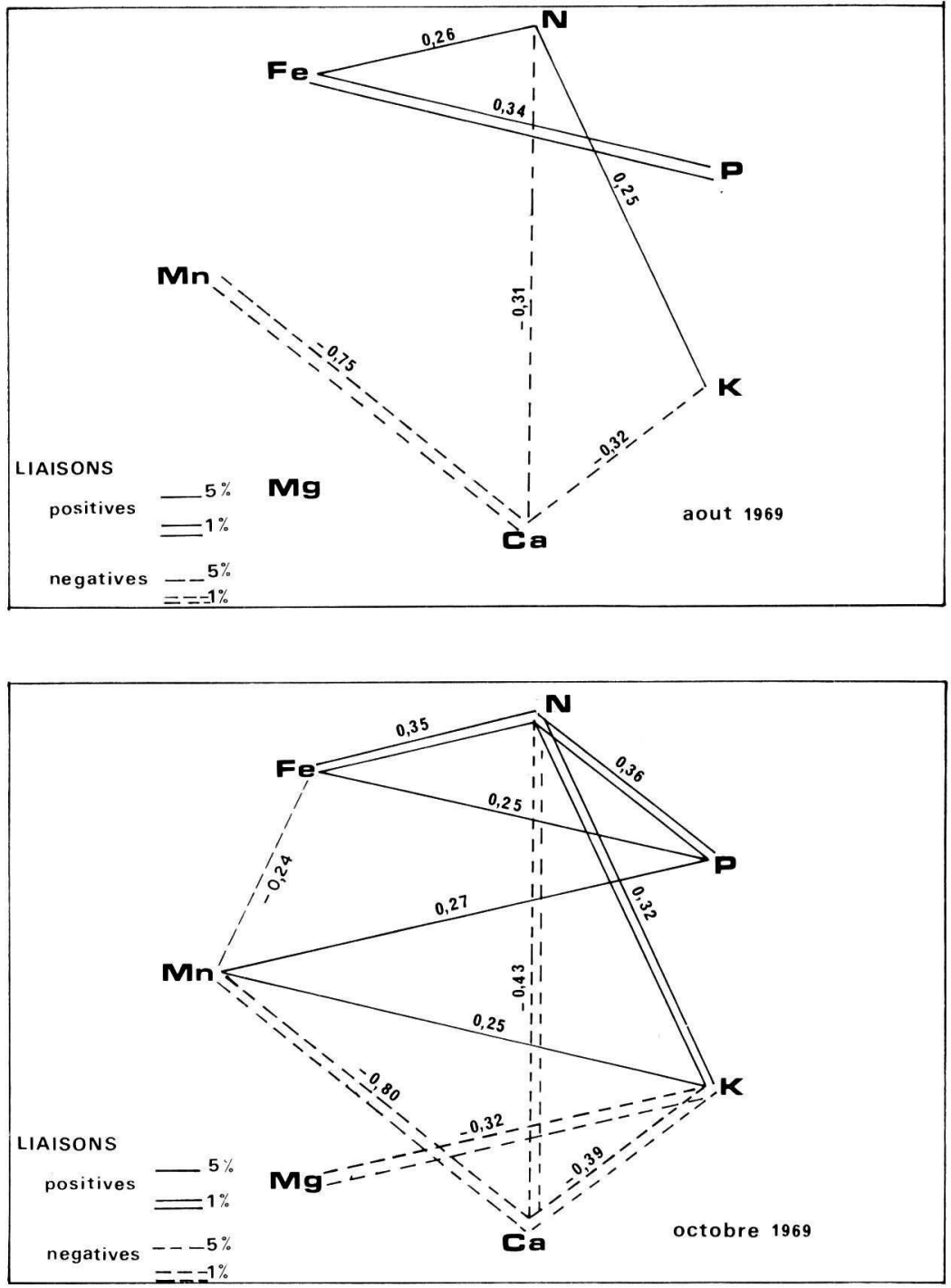

FIG. 13. - Liaison entre les différents éléments minéraux des feuilles de hêtre au mois d'août 1969 et au mois d'octobre 1969. (Seules les liaisons présentant un coefficient de corrélation significatif sont matérialisées).

$$
\begin{gathered}
n=65 \text { d.d.l. }=63 \\
r 5 \%=0,25 \\
r 1 \%=0,325
\end{gathered}
$$

FIG. 13. - Correlations between the different mineral elements of beech leaves in August 1969 and in October 1969 (only the significative correlations are mentionned)

$$
\begin{aligned}
& n=65 \text { d.d.l. }=63 \\
& \quad r 5 \%=0,25 \\
& r 1 \%=0,325
\end{aligned}
$$




\section{TABLEAU 6}

TABLE 6

Rapport entre la moyenne et l'écart type des teneurs en éléments minéraux des feuilles de hêtre d'une part au mois d'août et d'autre part au mois d'octobre (65 peuplements). Coefficients d'intercorrélation entre les deux dates de prélèvement.

Mineral composition of beech leaves in August and in October : Ratio between average and standard deviation in August and average and standard deviation in October. Correlation coefficients for each mineral element between August and October.

\begin{tabular}{c|c|c|c}
\hline Caractères & $\begin{array}{c}\text { moyenne d'octobre } \\
\text { moyenne d'août }\end{array}$ & $\begin{array}{c}\text { écart type d'octobre } \\
\text { écart type d'août }\end{array}$ & $\begin{array}{c}\text { Coefficient d'inter- } \\
\text { corrélation entre } \\
\text { août et octobre }\end{array}$ \\
\hline $\mathrm{N}$ & 0,46 & 1,34 & $0,611 \times \times$ \\
$\mathrm{P}$ & 0,56 & 1,37 & $0,882 \times \times$ \\
$\mathrm{K}$ & 0,70 & 0,83 & 0,255 \\
$\mathrm{Ca}$ & 1,21 & 1,38 & $0,927 \times \times$ \\
$\mathrm{Mg}$ & 0,77 & 1,75 & $0,429 \times \times$ \\
$\mathrm{Mn}$ & 1,51 & 1,62 & $0,975 \times \times$ \\
$\mathrm{Fe}$ & 1,75 & 1,89 & $0,550 \times \times$ \\
\hline \hline
\end{tabular}

Les caractères les plus reproductibles sont incontestablement le manganèse $(r=0,975)$, le calcium $(r=0,927)$ et le phosphore $(r=0,882)$. Pour ces trois éléments, bien que leur teneur en valeur absolue soit très différente en août et en octobre, le diagnostic c'est-à-dire le classement relatif des peuplements, sera exactement le même aux deux dates de prélèvement.

Les coefficients d'intercorrélation pour l'azote, le fer et le magnésium sont encore élevés. Cependant, ils nous paraissent encore trop faibles pour que la reproductibilité du diagnostic soit acceptable entre août et octobre.

Par contre, il n'existe pratiquement pas de corrélation entre la teneur en potassium du mois d'août et celle du mois d'octobre. Il n'y a par conséquent aucune reproductibilité du diagnostic entre août et octobre pour le potassium.

\section{III. - DISCUSSION}

La variabilité saisonnière des différents éléments minéraux des feuilles de hêtre est globalement assez semblable à celle que nous connaissons chez les autres feuillus étudiés jusqu'à présent (Chêne Leroy 1968, Peuplier Touzet et Heinrich 1970, Garbaye 1972). Cette étude met toutefois en évidence un certain nombre de phénomènes intéressants.

L'évolution au cours de la saison de végétation, de la teneur en eau des feuilles et de leur poids montre l'existence de trois phases principales : 
— une première phase de synthèse de matière carbonée, avec transfert d'éléments minéraux vers les feuilles.

- une deuxième phase qui débute au mois de juillet et se termine vers la fin août. Au cours de cette phase les phénomènes de synthèse foliaire et les phénomènes de migration vers d'autres organes tendent à s'équilibrer, au moins du point de vue pondéral.

- une troisième phase qui débute en septembre. Au cours de cette période il y a prédominance des phénomènes de transfert vers les zones productrices de bois ou de réserves sur les phénomènes de synthèse foliaire.

Cette évolution schématique globale recouvre des comportements spécifiques des divers éléments, qui peuvent suivre deux sortes d'évolution dans le temps : une évolution décroissante ou une évolution croissante.

L'azote, le potassium et le phosphore présentent une évolution décroissante, avec un palier pendant les mois de juillet et d'août. Le magnésium présente une évolution décroissante continue.

Une évolution inverse, sans palier aboutit à la concentration du calcium, du silicium, du manganèse et du fer en fin de période de végétation.

Ces types d'évolution ne semblent pas être remis en cause par l'intervention des facteurs secondaires tels que la qualité de feuilles d'ombre ou de lumière. Nous avons seulement pu noter, à l'inverse de ce qui a été observé chez les autres feuillus, en particulier chez le peuplier, que les feuilles d'ombre sont plus concentrées en éléments minéraux que les feuilles de lumière, sans que l'allure des courbes de variation soit modifiée.

L'étude de la variabilité stationnelle des éléments minéraux soulève un certain nombre de problèmes.

Il apparaît tout d'abord que pour l'ensemble des stations tous les éléments minéraux, sauf le potassium, sont beaucoup plus discriminants en automne qu'en été. Les graphiques de comparaison des deux stations extrêmes de l'étude saisonnière donnent d'ailleurs des courbes de variation généralement divergentes en automne, en particulier pour le calcium, le magnésium, le silicium et le fer.

- La relative constance de la teneur en azote des feuilles de stations à sol très différent est assez étonnante. En effet, la quantité d'azote minéralisé, comme la forme d'azote disponible sont très différentes entre les sols saturés ou calcaires et les sols désaturés (LE TACON 1972). Bien que certains auteurs aient montré une certaine corrélation en sol acide entre la production du hêtre et la nitrification (VAN PRAAG 1971), le hêtre semblerait manifester, pour une gamme très large de stations, une relative indifférence à la forme comme à la quantité d'azote disponible dans le sol. Cette propriété pourrait ainsi expliquer, au moins en partie, la très grande plasticité de cette essence.

Le rapport $\mathrm{C} / \mathrm{N}$ des feuilles prélevées sur un sol à mor et de celles prélevées sur rendzine est à peu près identique. Cela va de pair avec l'observation précédente, c'est-à-dire la faible variabilité de la teneur en azote des feuilles, surtout à leur chute, sur des stations pourtant très différentes.

Ces deux faits vont dans le sens des observations effectuées par l'un d'entre nous (Toutain et Duchaufour 1970), et s'opposent aux grandes différences de $\mathrm{C} / \mathrm{N}$ existant dans les pluviolessivats de hêtraie à mor et de hêtraie à mull (BRUCKERT et alt 1971). Il faut donc admettre que la variation de rapport $\mathrm{C} / \mathrm{N}$ des feuilles n'est pas une caractéristique de la sta- 
tion, et que la divergence du rapport $\mathrm{C} / \mathrm{N}$ qui aboutit au contraste des humus, se fait au niveau des pluviolessivats et surtout au niveau de la couche humifère (TOUTAIN 1972).

- C'est le manganèse qui discrimine le plus fortement l'ensemble des stations. En fin de saison de végétation le rapport entre les valeurs extrêmes est de 40 ! Le cycle biogéochimique du manganèse est particulièrement efficace. Il a un rôle essentiel dans la répartition du manganèse dans les profils pédologiques et accentue les écarts de teneurs observées dans les roches-mères (Vallée 1966, Vedy et Toutain 1971, Toutain 1972). Rappelons que les mulls acides favorisent l'accumulation du manganèse facilement réductible, alors que dans les moders et mors, le manganèse reste sous forme échangeable et est facilement lessivé. La teneur en manganèse des feuilles augmente donc lorsque l'on passe du mor au moder puis au mull moder. Avec les humus de type mull le calcium prend une importance considérable en freinant l'absorption du manganèse (antagonisme Ca-Mn) (LE TACON MiLlier 1970). Au fur et à mesure que le sol se sature en calcium, la teneur en manganèse diminue à nouveau. Enfin, dans les mulls calciques vient s'ajouter un phénomène de rétrogradation du manganèse, ce qui provoque une nouvelle diminution de la teneur en manganèse des feuilles.

Cet ensemble de phénomène permet d'expliquer pourquoi les écarts observés entre les deux stations extrêmes, La Costelle (mor) et de Haye (mull calcique) ne sont pas très importants.

- Le comportement du potassium est très particulier. Il n'existe aucune reproductibilité entre le diagnostic établi au mois d'août, et celui établi au mois d'octobre pour le potassium, alors que la reproductibilité est bonne pour tous les autres éléments et parfois même remarquable (manganèse, calcium, phosphore). Le potassium dans les tissus n'est pas engagé comme le calcium, le manganèse ou le phosphore dans des complexes. Il existe essentiellement sous forme de sels solubles et son évolution dépend non seulement des mécanismes physiologiques, mais aussi des pertes par pluviolessivage (VEDY DUCHAUfour 1970, Clément 1972). En fin de saison de végétation les teneurs en potassium des feuilles sont donc plus sous la dépendance du pluviolessivage que des caractéristiques chimiques des stations.

\section{CONCLUSION}

Cette étude a permis de préciser de nombreux points concernant la nutrition minérale du hêtre et d'apporter quelques nouveaux éléments concernant les cycles biogéochimiques.

Du point de vue méthodologique, en vue de l'établissement d'un diagnostic foliaire, il apparaît qu'à la chute des feuilles, la discrimination entre stations de productivité bien différente est maximale pour toutes les caractéristiques étudiées, hormis le potassium. La teneur en potassium dans les feuilles, à l'automne, semble en effet sous la dépendance du pluviolessivage et non des caractéristiques de la station.

Le manque de fidélité du diagnostic pour cet élément en octobre semble suffisant pour nous inciter à adopter une méthode de prélèvement estival. Le mois d'août correspond en effet très nettement à une période d'équilibre entre les phénomènes de synthèse dans les feuilles et les phénomènes de transfert vers les organes non chlorophylliens.

L'étude de la variation stationnelle nous permet, en outre, en première approximation de donner des valeurs optimales pour les éléments principaux. 
Des données beaucoup plus précises seront fournies par les résultats des essais de fertilisation actuellement en cours.

D'un point de vue plus fondamental cette note apporte une confirmation d'études antérieures (Duchaufour 1968, Souchier 1971, Toutain 1972, Vedy et alt 1971), qui ont mis en relief le rôle respectif du cycle biogéochimique et de la roche-mère dans l'évolution des horizons organiques des sols forestiers. Les faibles différences du rapport $\mathrm{C} / \mathrm{N}$ des feuilles entre des stations extrêmes à mor et à mull calcique oblige à admettre que cette valeur n'est pas pour l'essence étudiée une caractéristique de la station.

La divergence des rapports $\mathrm{C} / \mathrm{N}$ des types d'humus se fait ultérieurement au niveau de la couche humifère (TouTAIN 1972). II faut noter la haute signification de la teneur des feuilles en éléments tels que le fer ou la manganèse dans la discrimination des stations et la faible discrimination de la teneur en azote. Ces deux éléments, fer et manganèse, à signification directe peu importante dans le diagnostic de nutrition, pourraient être déterminants dans l'explication de la divergence des types d'humus. En effet, s'ils ont, en tant qu'éléments constitutifs de la roche-mère une très grande influence sur la formation des types d'humus en milieu acide (Souchier 1971, Toutain 1972), ils pourraient déjà, dans la litière, jouer un grand rôle dans l'évolution de la matière organique hydrosoluble.

Reçu pour publication en janvier 1973.

\section{SUMMARY}

VARIATIONS OF THE Mineral LeVEl IN BEeCh (Fagus Sylvatica) LEAVES IN THE EASTERn PART OF FRANCE ACCORDING TO SEASON AND SITE

This work shows that discrimination between sites is the best with falling leaves in autumn for all foliar characteristics, except potassium. Potassium level in leaves seems to be more dependant of leaching by rain, than of site in autumn.

So, we prefer summer sampling for foliar diagnostic (August). In August, indeed, there is a balance between foliar synthesis and translocation into wood.

The study of mineral elements concentration of beech leaves in 65 sites gives us optimal values for macroelements concentration ( $\mathrm{N}, \mathrm{P}, \mathrm{K}$ ).

More precise data will be given after results of different fertilization experiments we have settled.

In a more fundamental point of view, this paper confirms precedent studies (Duchaufour 1968, SOUCHIER 1971, VEDY et alt 1971, TOutAIN 1972) which have shown the importance of mineral cycle and of parent rock on humus evolution.

The low differences of leaves $\mathrm{C} / \mathrm{N}$ ratio between extreme sites (mor and calcic mull) show that this ratio is not characteristic of the site. The $\mathrm{C} / \mathrm{N}$ ratio becomes different later, during humification.

We must emphasize the importance of $\mathrm{Fe}$ and $\mathrm{Mn}$ in discriminating sites, and the low discrimination of $\mathrm{N}$.

These two elements, Fe and Mn, of small importance in nutrition diagnostic, could determine the different humus types, as other parent rock characteristics (calcium or clay content). SouchiER (1971) has already proposed the something for Fe.

\section{ZUSAMMENFASSUNG}

JAHRESZEITLICHE UND STANDORTLICHE SCHWANKUNGEN DES NÄHRSTOFFSPIEGELS DER ROTBUCHE (Fagus sylvatica)

IN OSTFRANKREICH

Die vorliegende Untersuchung zeigt deutlich, dass die im Herbst bestimmten Nährstoffspiegelwerte - mit Ausnahme der Kaliwerte - eine sehr gute Unterscheidung der Buchenstandorte ermöglichen. 
Der Kaligehalt der Blätter im Herbst scheint mehr von der Auswaschung durch die Niederschläge als von den Standortmerkmalen beeinflusst.

Wir haben daher den August für die Bestimmung der Nährstoffspiegelwerte vorgezogen. Dieser Zeitpunkt entspricht einer Stabilitätsperiode, während welcher ein Gleichgewicht zwischen der Nährstoffsynthese und der Migration in die Holzproduktionsorgane vorzuliegen scheint.

Die Bestimmung der Nährstoffspiegelwerte an 65 Buchenstandorten erlaubte erste Aussagen über Optimalwerte an N, P und K im August. Eine genauere Bestimmung der Optimalwerte erfolgt nach Auswertung der zur Zeit laufenden Düngeversuche.

Die vorliegende umfassende Arbeit bestätigt die Ergebnisse früherer Untersuchungen anderer Autoren (Duchaufour 1968, SouChIER 1971, Vedy et al. 1971, Toutain 1972), welche die Bedeutung der Mineralnährstoffzyklen und des Grundgesteins bezüglich der Humusevolution hervorhoben.

Die geringen Unterschiede im C/N Verhältnis der Blätter von sehr unterschiedlichen Standorten (Kalkmulloder Moder), zeigt, dass dieser Wert zur Unterscheidung der Standorte schlecht geeignet ist. Das C/N Verhältnis wird erst später, im Laufe der Humifizierung sehr unterschiedlich.

Die Stickstoffwerte der Blätter zeigen nur ein geringes Trennvermögen, während der Gehalt an Eisen und Mangan wesentlich zur Differenzierung der Standorte beiträgt. Diese beiden Elemente, welche für die Ernährungsdiagnostik nur eine geringe Bedeutung haben, dürften jedoch bei der Bildung der verschiedenen Humustypen gleichrangig mit anderen Merkmalen des Grundgesteins (Tonund Kalziumgehalt) eine wichtige Rolle spielen.

SOUCHIER (1971) hat diese Hypothese bereits für den Eisengehalt vorgeschlagen.

\section{RÉFÉRENCES BIBLIOGRAPHIQUES}

Bruckert S., Toutain F., Tchikaya J. et JaCquin F., 1971. - Influence des pluviolessivats de Hêtre et de Pin Sylvestre sur les processus d'humification. Oecologia Plantarum, 6, 329-339, Gauthier Villars.

Clément A. 1972. - Étude de l'équilibre acido-basique de l'Épicéa sur sol très carbonaté, carbonaté et décarbonaté. Thèse, Fac. Sciences, Nancy.

DAgnelie P., 1956. - Recherches sur la productivité des hêtraies d'Ardenne en relation avec les types phytosociologiques et les facteurs écologiques. $1^{\text {re }}$ partie - Recherche d'un critère de station utilisable dans les hêtraies d'Ardenne. Bull. Inst. Agron. Stat. Rech. Gembl. 24, 3, 249-284.

DAGNELIE P., 1956. - Recherche sur la productivité des hêtraies d'Ardenne en relation avec les types phytosociologiques et les facteurs écologiques. 2e partie - Utilisation d'un critère de station dans les hêtraies d'Ardenne. Bull. Inst. Agron. Stat. Rech. Gembl., 24, 4, 349-410.

Dagnelie P., 1957. - Recherches sur la productivité des hêtraies d'Ardenne en relation avec les types phytosociologiques et les facteurs écologiques. $3^{\circ}$ partie - Interprétation des résultats Bull. Inst. Agron. Stat. Rech. Gembl., 25, 1-2, 44-94.

Duchaufour Ph., 1968. - L'évolution des sols - essai sur la dynamique des profils. Masson et Cie édit. Paris.

Galoux A., 1954. - Phytosociologie et applications sylvicoles. VIII congrès international de Botanique, Paris, $31-34$.

Garbaye J., 1972. - L'influence de la date et de la hauteur de prélèvement sur les résultats de l'analyse foliaire chez deux clônes de peuplier. Ann. Sciences forest. (sous presse).

Guilimondi G., 1960. - Richerch preliminari sulla nutrizione minerale del pioppo a nezzo dell'analisi fogliare. Pubbl. Centre sper. agric. for., 4, 231-245.

Guha M., Mitchell R.L.V., 1966. - The trace and major element composition of the leaves of some deciduous trees seasonal changes. Plant and Soil, 24, 90-112.

Leroy P., 1968. - Variations saisonnières des teneurs en eau et en éléments minéraux des feuilles de chêne (Quercus pedunculata). Ann. Sciences forestières, 25 (2) 83-117.

Le TACON F., Millier C., 1970. — La nutrition minérale de l'Épicéa commun en sols carbonatés et en sols décarbonatés, essai sur le comportement du calcaire et du manganèse. Ann. Sci. Forest., 27 (1) $63-88$.

LE TACON F., 1972. - Disponibilité de l'azote nitrique et ammoniacal dans certains sols de l'Est de la France. Influence sur la nutrition et la croissance de l'Épicéa commun. Ann. Sci. forest., 30 (2) 183-207.

Manil G., Delecour F., Forget G., El Attar A., 1963. - L'humus facteur de station dans les hêtraies acidophiles de Belgique. Bulletin de l'Institut agronomique et des Stations de Recherches de Gembloux. T. XXXI nos 1 et 2, 1 à 114. 
Polge., Keller., Thiercelin., 1972. - Effets du sol et de l'hérédité sur la croissance et les caractéristiques de jeunes plants de Hêtre. I.N.R.A. Centre National de Recherches Forestières, Nancy Distribution limitée.

Reginster P., 1955. - La productivité stationnelle des hêtraies d'Ardenne. Bulletin de la Société royale forestière de Belgique, $\mathrm{n}^{\circ}$ 1, 1-8.

Souchier B. 1971. - Évolution des sols sur roches cristallines à l'étage montagnard (Vosges) Thèse, i. Faculté des Sciences de Nancy.

Toutain F., Duchaufour Ph., 1970. - Étude comparée des bilans biologiques de certains sols de hêtraie. Ann. Sci. Forestières, (1) 27 39-61.

Toutain F., 1972. - Étude comparée de deux hêtraies sur grès rhétien : divergences pédoclimatiques et biochimiques. Bull. E.N.S.A.I.A. Nancy, T. XIV, fasc. 1.

Touzet G., Heinrich J.-C., 1970. - Concentration foliaire en azote, phosphore, potassium et calcium du Peuplier. C.V.I. 214. A.F.O.C.E.L.

VALléE G., 1967. - Étude du rôle du manganèse dans la régénération de la sapinière vosgienne. Ann. Sciences forestières, 24 (4) 259-275.

Van Praag H. J., - Contribution à l'étude de la disponibilité de l'azote, et du soufre dans les sols forestiers oligotrophes de l'Ardenne. Thèse, Fac. Sciences Agronomiques Gembloux, Belgique.

Vedy J.-C., Duchaufour Ph., 1971. - Cycle biogéochimique du potassium dans les premières phases de la pédogénèse en milieu acide. C. R. Acad. Sc. Paris, 272, 1598-1601 (série D).

Vedy J.-C., Toutain F., 1971. - Cycle biogéochimique du manganèse en milieu acide. C. R. Acad. Paris, T. 273, 136-139 (série D). 